MATHEMATICS OF COMPUTATION

Volume 80, Number 274, April 2011, Pages 905-930

S $0025-5718(2010) 02433-0$

Article electronically published on November 2, 2010

\title{
EXPONENTIAL CONVERGENCE AND TRACTABILITY OF MULTIVARIATE INTEGRATION FOR KOROBOV SPACES
}

\author{
JOSEF DICK, GERHARD LARCHER, FRIEDRICH PILLICHSHAMMER, \\ AND HENRYK WOŹNIAKOWSKI
}

\begin{abstract}
In this paper we study multivariate integration for a weighted Korobov space for which the Fourier coefficients of the functions decay exponentially fast. This implies that the functions of this space are infinitely times differentiable. Weights of the Korobov space monitor the influence of each variable and each group of variables. We show that there are numerical integration rules which achieve an exponential convergence of the worst-case integration error. We also investigate the dependence of the worst-case error on the number of variables $s$, and show various tractability results under certain conditions on weights of the Korobov space. Tractability means that the dependence on $s$ is never exponential, and sometimes the dependence on $s$ is polynomial or there is no dependence on $s$ at all.
\end{abstract}

\section{INTRODUCTION}

Multivariate integration of $s$-variate functions is a popular research subject especially the case when the number of variables $s$ is large. We usually want to find the best possible rate of convergence as well as to control the dependence on $s$. The latter problem is related to tractability when we want to guarantee that there is no exponential dependence on $s$.

In this paper we study the numerical approximation of integrals

$$
\int_{[0,1]^{s}} f(\boldsymbol{x}) \mathrm{d} \boldsymbol{x}
$$

using quasi-Monte Carlo rules

$$
\frac{1}{n} \sum_{m=1}^{n} f\left(\boldsymbol{t}_{m}\right)
$$

Here, the quadrature points $\boldsymbol{t}_{1}, \boldsymbol{t}_{2}, \ldots, \boldsymbol{t}_{n} \in[0,1]^{s}$ are chosen in some deterministic way. In our case, $\boldsymbol{t}_{i}$ will be defined by lattice rules of rank one or will be from grids with varying mesh-sizes. For more information on such quadrature rules see e.g., 10, 14.

Received by the editor July 13, 2009 and, in revised form, March 17, 2010.

2010 Mathematics Subject Classification. Primary 11K45, 65C05, 65D30.

Key words and phrases. Quasi-Monte Carlo, numerical integration, lattice rules, tractability.

The second author was supported by the Austrian Science Foundation (FWF), Project P21196.

The third author was supported by the Austrian Research Foundation (FWF), Project S 9609, which is part of the Austrian Research Network "Analytic Combinatorics and Probabilistic Number Theory".

The fourth author was partially supported by the National Science Foundation. 
We assume that the integrands $f$ are periodic and have a Fourier series representation

$$
f(\boldsymbol{x})=\sum_{\boldsymbol{h} \in \mathbb{Z}^{s}} \widehat{f}(\boldsymbol{h}) \exp (2 \pi \mathrm{i} \boldsymbol{h} \cdot \boldsymbol{x}),
$$

where the Fourier coefficients $\widehat{f}$ are given by

$$
\widehat{f}(\boldsymbol{h})=\int_{[0,1]^{s}} f(\boldsymbol{x}) \exp (-2 \pi \mathrm{i} \boldsymbol{h} \cdot \boldsymbol{x}) \mathrm{d} \boldsymbol{x} .
$$

The smoothness of the integrand $f$ is regulated by the decay of its Fourier coefficients. Here we assume that the Fourier coefficients decay exponentially fast, i.e.,

$$
\widehat{f}(\boldsymbol{h})=\mathcal{O}\left(\omega^{\left|h_{1}\right|+\cdots+\left|h_{s}\right|}\right),
$$

where $\boldsymbol{h}=\left(h_{1}, \ldots, h_{s}\right) \in \mathbb{Z}^{s}$ and $0<\omega<1$.

This corresponds to the Korobov space of infinitely times differentiable functions. This is a reproducing kernel Hilbert space with the explicitly known kernel. We study the unweighted case for which all variables and groups of variables are equally important, as well as the weighted case for which the influence of each variable and each group of variables is moderated by a suitable weight. We show that the rate of convergence is independent of weights whereas tractability results are possible only for properly decaying weights.

Previously, numerical integration of periodic functions has been analyzed for functions which are $\alpha$ times differentiable in each variable with $\alpha<\infty$; see for example [6, 17, 12, 15, 18. Our approach for infinitely times differentiable functions is similar to the approach in those papers. Indeed, we also define a suitable reproducing kernel Hilbert space, although the analysis of the worst-case integration error turns out to be somewhat different than in the papers cited above.

We show the existence of lattice rules which achieve an exponential convergence (Theorems 2 and 4). This is done by defining a suitable figure of merit and proving results on the existence of lattice rules with a large figure of merit (Lemma 2). A lower bound on the worst-case error reveals that this rate of convergence is essentially best possible. The upper bound is nonconstructive, as the proof only shows the existence of suitable lattice rules. We do, however, provide "more" constructive results. To be more precise, we show how a suitable generating vector can be found explicitly in some sense (Remark 1). We also show that a quasi-Monte Carlo rule with the quadrature points from a grid with suitable varying mesh-sizes achieves an exponential rate of convergence (Theorem [6).

We show that the trigonometric degree of a lattice rule [3, 4, 8, 13, plays an important role in our study. Indeed, for the unweighted case, the figure of merit which is used for proving existence results for lattice rules achieving an exponential convergence coincides with their enhanced trigonometric degree. Hence, lattice rules with high trigonometric degree are needed for achieving exponential rates of convergence for integration in the Korobov space.

We also study how the worst-case error depends on the number of variables $s$. For the unweighted case, despite the fact that the functions are infinitely times differentiable, we still get an exponential increase of the worst-case error with growing $s$ (Theorem 3). This situation can only be remedied by changing to the weighted case (Theorems 5 and 6 and Corollary 1). This is done by introducing a weighted 
version of the Korobov space, an idea which stems from [17]. It is, however, necessary to change the dependence on the different coordinate directions in a different way compared to [17] if one wants to have both, independence of the dimension and an exponential convergence of the worst-case error. Only if one is satisfied with a polynomial rate of convergence of the form $n^{-\alpha}$, where $\alpha>0$ can be arbitrarily large, the weights defined as in [17. suffice to guarantee strong polynomial tractability (which is the technical term for the worst-case error being bounded independent of the dimension, see below for details) (Remark 3). If one wants both, an exponential convergence and strong polynomial tractability, then we need to weight the variables in a different way. We also investigate under which conditions on the weights we obtain polynomial tractability.

\section{Definition of the PRoblem}

We consider a Korobov space $H(K)$ of complex-valued periodic functions defined on $[0,1]^{s}$. The space $H(K)$ is a reproducing kernel Hilbert space with the kernel

$$
K(\boldsymbol{x}, \boldsymbol{y})=\sum_{\boldsymbol{h} \in \mathbb{Z}^{s}} \omega_{\boldsymbol{h}} \exp (2 \pi \mathrm{i} \boldsymbol{h} \cdot(\boldsymbol{x}-\boldsymbol{y})) \quad \text { for all } \boldsymbol{x}, \boldsymbol{y} \in[0,1]^{s},
$$

with the usual inner product $\boldsymbol{h} \cdot(\boldsymbol{x}-\boldsymbol{y})=\sum_{j=1}^{s} h_{j}\left(x_{j}-y_{j}\right)$, where $h_{j}, x_{j}, y_{j}$ are the $j$ th components of the vectors $\boldsymbol{h}, \boldsymbol{x}, \boldsymbol{y}$, correspondingly. (For information about reproducing kernel Hilbert spaces we refer to [1.)

We assume that $\omega_{\mathbf{0}}=1$ and $\omega_{\boldsymbol{h}}$ may also depend on $s$, i.e., $\omega_{\boldsymbol{h}}=\omega_{s, \boldsymbol{h}}$, and is nonnegative for all $\boldsymbol{h} \in \mathbb{Z}^{s}$. The kernel $K$ is well defined if we choose $\omega_{\boldsymbol{h}}$ such that

$$
|K(\boldsymbol{x}, \boldsymbol{y})| \leq K(\boldsymbol{x}, \boldsymbol{x})=\sum_{\boldsymbol{h} \in \mathbb{Z}^{s}} \omega_{\boldsymbol{h}}<\infty .
$$

For $f \in H(K)$ we have

$$
f(\boldsymbol{x})=\sum_{\boldsymbol{h} \in \mathbb{Z}^{s}} \widehat{f}(\boldsymbol{h}) \exp (2 \pi i \boldsymbol{h} \cdot \boldsymbol{x}) \quad \text { for all } \quad \boldsymbol{x} \in[0,1]^{s},
$$

and the norm of $f$ from $H(K)$ is given in terms of its Fourier coefficients $\widehat{f}$ by

$$
\|f\|^{2}=\sum_{\boldsymbol{h} \in \mathbb{Z}^{s}}|\widehat{f}(\boldsymbol{h})|^{2} \omega_{\boldsymbol{h}}^{-1}<\infty .
$$

The inner product of $f$ and $g$ from $H(K)$ is

$$
\langle f, g\rangle=\sum_{\boldsymbol{h} \in \mathbb{Z}^{s}} \widehat{f}(\boldsymbol{h}) \overline{\widehat{g}(\boldsymbol{h})} \omega_{\boldsymbol{h}}^{-1} .
$$

Smoothness of functions $f$ from $H(K)$ is controlled by how fast $\omega_{\boldsymbol{h}}$ goes to zero as $|\boldsymbol{h}|:=\sum_{j=1}^{s}\left|h_{j}\right|$ goes to infinity. We assume that there exists $\omega \in(0,1)$ such that

$$
\omega_{\boldsymbol{h}}=\mathcal{O}\left(\omega^{|\boldsymbol{h}|}\right) \quad \text { for all } \quad \boldsymbol{h}=\left(h_{1}, h_{2}, \ldots, h_{s}\right) \in \mathbb{Z}^{s} .
$$

Then functions from $H(K)$ are infinitely times differentiable. Indeed, for $f \in$ $H(K)$, let $\boldsymbol{\alpha}=\left(\alpha_{1}, \alpha_{2}, \ldots, \alpha_{s}\right)$ be an arbitrary vector with integers $\alpha_{j} \geq 0$. Denote by

$$
D^{\boldsymbol{\alpha}} f=\frac{\partial^{|\boldsymbol{\alpha}|}}{\partial x_{1}^{\alpha_{1}} \partial x_{2}^{\alpha_{2}} \cdots \partial x_{s}^{\alpha_{s}}} f
$$


the operator of partial differentiation. Then

$$
D^{\boldsymbol{\alpha}} f(\boldsymbol{x})=\sum_{\boldsymbol{h} \in \mathbb{Z}^{s}}\left[\widehat{f}(\boldsymbol{h})(2 \pi \mathrm{i})^{|\boldsymbol{\alpha}|} \prod_{j=1}^{s} h_{j}^{\alpha_{j}}\right] \exp (2 \pi \mathrm{i} \boldsymbol{h} \cdot \boldsymbol{x}),
$$

where, by convention, we take $0^{0}=1$. The last series is convergent. Indeed, for any $\omega_{1} \in(\omega, 1)$ there is a number $C$ depending on $\omega, \omega_{1}$ and $|\boldsymbol{\alpha}|$ such that

$$
x^{a} \omega^{x} \leq C \omega_{1}^{x} \text { for all } a \in[0,2|\boldsymbol{\alpha}|] \text { and } x \in[0, \infty) .
$$

Then $\omega_{\boldsymbol{h}}=\mathcal{O}\left(\omega^{|\boldsymbol{h}|}\right)$ implies

$$
\begin{aligned}
\left|D^{\boldsymbol{\alpha}} f(\boldsymbol{x})\right| & =\left|\sum_{\boldsymbol{h} \in \mathbb{Z}^{s}}\left[\widehat{f}(\boldsymbol{h}) \omega_{\boldsymbol{h}}^{-1 / 2}\right]\left[\omega_{\boldsymbol{h}}^{1 / 2}(2 \pi \mathrm{i})^{|\boldsymbol{\alpha}|} \prod_{j=1}^{s} h_{j}^{\alpha_{j}}\right] \exp (2 \pi \mathrm{i} \boldsymbol{h} \cdot \boldsymbol{x})\right| \\
& =\mathcal{O}\left(\|f\|\left[\sum_{\boldsymbol{h} \in \mathbb{Z}^{s}}(2 \pi)^{2|\boldsymbol{\alpha}|} \prod_{j=1}^{s}\left|h_{j}\right|^{2 \alpha_{j}} \omega^{\left|h_{j}\right|}\right]^{1 / 2}\right) \\
& =\mathcal{O}\left(\|f\|\left[\sum_{\boldsymbol{h} \in \mathbb{Z}^{s}} \omega_{1}^{|\boldsymbol{h}|}\right]^{1 / 2}\right) \\
& =\mathcal{O}\left(\|f\|\left(1+\frac{2}{1-\omega_{1}}\right)^{s / 2}\right)<\infty
\end{aligned}
$$

as claimed.

Consider multivariate integration,

$$
I(f)=\int_{[0,1]^{s}} f(\boldsymbol{x}) \mathrm{d} \boldsymbol{x} \text { for all } f \in H(K) .
$$

The problem is well normalized since

$$
\|I\|=\sup _{f \in H(K),\|f\| \leq 1}\left|\int_{[0,1]^{s}} f(\boldsymbol{x}) \mathrm{d} \boldsymbol{x}\right|=1 .
$$

We approximate $I(f)$ by algorithms that use finitely many function values. It is known that we can restrict ourselves to linear algorithms and approximate $I(f)$ by

$$
A_{n, s}(f)=\sum_{m=1}^{n} a_{m} f\left(\boldsymbol{t}_{m}\right)
$$

for some complex numbers $a_{m}$ and sample points $\boldsymbol{t}_{m} \in[0,1]^{s}$. For $a_{m}=n^{-1}$, we obtain popular quasi-Monte Carlo (QMC) algorithms that are often used in computational practice, especially for large $s$. By

$$
e^{\text {wor }}\left(A_{n, s}\right)=\sup _{f \in H(K),\|f\| \leq 1}\left|I(f)-A_{n, s}(f)\right|
$$

we mean the worst-case error of $A_{n, s}$. Since $I(f)=\langle f, 1\rangle$, then

$$
I(f)-A_{n, s}(f)=\left\langle f, 1-\sum_{m=1}^{n} a_{m} K\left(\cdot, \boldsymbol{t}_{m}\right)\right\rangle .
$$


Therefore,

$$
e^{\mathrm{wor}}\left(A_{n, s}\right)=\left\|1-\sum_{m=1}^{n} a_{m} K\left(\cdot, \boldsymbol{t}_{m}\right)\right\|
$$

This can be easily computed to be

$$
\left[e^{\text {wor }}\left(A_{n, s}\right)\right]^{2}=1-2 \sum_{m=1}^{n} a_{m}+\sum_{k, m=1}^{n} a_{k} a_{m} K\left(\boldsymbol{t}_{k}, \boldsymbol{t}_{m}\right) .
$$

For QMC algorithms, $a_{m}=n^{-1}$, the last formula simplifies to

$$
\left[e^{\mathrm{wor}}\left(A_{n, s}\right)\right]^{2}=-1+\frac{1}{n^{2}} \sum_{k, m=1}^{n} K\left(\boldsymbol{t}_{k}, \boldsymbol{t}_{m}\right) .
$$

Let $e(n, s)$ be the $n$th minimal worst-case error,

$$
e(n, s)=\inf _{a_{j}, \boldsymbol{t}_{j}, j=1,2, \ldots, n} \sup _{f \in H(K),\|f\| \leq 1}\left|I(f)-\sum_{m=1}^{n} a_{m} f\left(\boldsymbol{t}_{m}\right)\right| .
$$

For $n=0$ we approximate $I(f)$ simply by zero, and $e(0, s)=\|I\|=1$ for all $s$.

What do we want to demand on the behavior of $e(n, s)$ to capture the notion of exponential convergence and tractability?

By exponential convergence we mean that there exist numbers $q \in(0,1), p>0$ and a function $C: \mathbb{N} \rightarrow(0, \infty)$ such that

$$
e(n, s) \leq C(s) q^{n^{p}} \quad \text { for all } \quad s, n \in \mathbb{N} \text {. }
$$

Let

$$
n(\varepsilon, s)=\min \{n: e(n, s) \leq \varepsilon\}
$$

be the minimal number of function values needed to obtain an $\varepsilon$ approximation.

If (3) holds, then

$$
n(\varepsilon, s) \leq\left\lceil\left(\frac{\ln C(s)+\ln \varepsilon^{-1}}{\ln q^{-1}}\right)^{1 / p}\right\rceil \quad \text { for all } \quad s \in \mathbb{N}, \varepsilon \in(0,1) .
$$

Observe that if (4) holds, then

$$
e(n+1, s) \leq C(s) q^{n^{p}} \quad \text { for all } \quad s, n \in \mathbb{N} .
$$

This means that (3) and (44) are essentially equivalent.

Hence, exponential convergence implies that asymptotically with respect to $\varepsilon$, we need to perform $\mathcal{O}\left(\left[\ln \varepsilon^{-1}\right]^{1 / p}\right)$ function values to compute an $\varepsilon$ approximation to multivariate integrals. However, it is not clear how long we have to wait to see this nice asymptotic behavior especially for large $s$. This, of course, depends on $C(s)$ and is the subject of tractability. Tractability means that we control the behavior of $C(s)$ and rule out the cases for which $n(\varepsilon, s)$ depends exponentially on $s$. Since there are many ways of controlling the lack of exponential dependence, we have many notions of tractability. We restrict ourselves to two such notions in this paper; for more on tractability see [11].

We say that we have exponential convergence with polynomial tractability iff there exist nonnegative numbers $A, p_{1}, p_{2}$ such that

$$
n(\varepsilon, s) \leq A\left(s^{p_{1}}+\left[\ln \varepsilon^{-1}\right]^{p_{2}}\right) \quad \text { for all } \quad s \in \mathbb{N}, \varepsilon \in(0,1) .
$$


If $p_{1}=0$, we say that we have exponential convergence with strong polynomial tractability.

We say that we have exponential convergence with weak tractability 1 iff

$$
\lim _{\varepsilon^{-1}+s \rightarrow \infty} \frac{\ln n(\varepsilon, s)}{\ln \varepsilon^{-1}+s}=0 .
$$

In this paper we always consider exponential convergence, and therefore if we say (strong) polynomial tractability or weak tractability we always mean exponential convergence with (strong) polynomial tractability or with weak tractability. Obviously, polynomial tractability implies weak tractability.

Assume that (3) is satisfied. Then (strong) polynomial tractability holds if

$$
\sup _{s \in \mathbb{N}} s^{-\tau} \ln (1+C(s))<\infty \quad \text { for some } \quad \tau \geq 0 .
$$

If so, then we have (strong) polynomial tractability with $p_{1}=\tau / p$ and $p_{2}=1 / p$.

Weak tractability holds if

$$
\lim _{s \rightarrow \infty} \frac{\ln (1+\ln (1+C(s)))}{s}=0 .
$$

Hence, strong polynomial tractability holds if $C(s)$ are uniformly bounded in $s$, polynomial tractability holds if there exist nonnegative numbers $A$ and $\tau$ such that

$$
C(s) \leq \exp \left(A s^{\tau}\right) \text { for all } s \in \mathbb{N},
$$

and weak tractability holds if

$$
C(s)=\exp (\exp (o(s))) \quad \text { as } s \rightarrow \infty .
$$

The conditions on $C(s)$ seem to be quite weak since even for singly exponential $C(s)$ we have polynomial tractability, and for "almost" doubly exponential $C(s)$ we have weak tractability. We shall see later for which $\omega_{\boldsymbol{h}}$ we can indeed guarantee (strong) polynomial and weak tractability.

We also add that if (3) is sharp, i.e., there exists a positive number $C$ independent of $n$ and $s$ such that

$$
e(n, s) \geq C C(s) q^{n^{p}} \quad \text { for all } \quad s, n \in \mathbb{N},
$$

then the conditions on $C(s)$ presented above are also necessary.

\section{LOWER BOUND}

We study multivariate integration for Korobov spaces for different choices of $\omega_{\boldsymbol{h}}$. All of our choices of $\omega_{\boldsymbol{h}}$ will lead to the exponential convergence or to an almost exponential convergence. However, tractability will hold only for some choices of $\omega_{\boldsymbol{h}}$. In this short section we establish a lower bound on $e(n, s)$ in terms of $\omega_{\boldsymbol{h}}$ that will allow us later to verify tractability for specific choices of $\omega_{\boldsymbol{h}}$. The idea of the proof of this lower bound is adopted from [16].

Let $k$ and $t$ be positive integers. For $s \geq k$, define the set

$$
\mathcal{A}_{s, k, t}=\left\{\boldsymbol{h} \in \mathbb{Z}^{s}: s-k \text { of } h_{j} \text { are } 0 \text { and } k \text { of } h_{j} \text { are from }\{1,2, \ldots, t\}\right\} .
$$

${ }^{1}$ Weak tractability without specifying the rate of convergence is defined as

$$
\lim _{\varepsilon^{-1}+s \rightarrow \infty} \frac{\ln n(\varepsilon, s)}{\varepsilon^{-1}+s}=0 .
$$

Hence, exponential convergence with weak tractability differs from weak tractability in the role of $\varepsilon$. 
The cardinality of $\mathcal{A}_{s, k, t}$ is clearly $\left(\begin{array}{l}s \\ k\end{array}\right) t^{k}$.

Theorem 1. Let $k$ and $t$ be positive integers. With the notation from above, the nth minimal worst-case error satisfies

$$
e(n, s) \geq\left[\max _{\boldsymbol{h}^{*} \in \mathcal{A}_{s, k, t}} \sum_{\boldsymbol{h} \in \mathcal{A}_{s, k, t}} \frac{1}{\omega_{\boldsymbol{h}-\boldsymbol{h}^{*}}}\right]^{-1 / 2} \quad \text { for all } n<\left(\begin{array}{l}
s \\
k
\end{array}\right) t^{k} .
$$

Proof. Take an arbitrary algorithm $A_{n, s}(f)=\sum_{m=1}^{n} a_{m} f\left(\boldsymbol{t}_{m}\right)$. Define

$$
g(\boldsymbol{x})=\sum_{\boldsymbol{h} \in \mathcal{A}_{s, k, t}} b_{\boldsymbol{h}} \exp (2 \pi \mathrm{i} \boldsymbol{h} \cdot \boldsymbol{x}) \quad \text { for all } \quad \boldsymbol{x} \in[0,1]^{d}
$$

such that $g\left(\boldsymbol{t}_{m}\right)=0$ for all $m=1,2, \ldots, n$. Since we have $n$ homogeneous linear equations and $\left|\mathcal{A}_{s, k, t}\right|>n$ unknowns $b_{\boldsymbol{h}}$, there exists a nonzero vector of such $b_{\boldsymbol{h}}$ 's, and we can normalize the $b_{\boldsymbol{h}}$ 's by assuming that

$$
\max _{\boldsymbol{h} \in \mathcal{A}_{s, k, t}}\left|b_{\boldsymbol{h}}\right|=b_{\boldsymbol{h}^{*}}=1 \quad \text { for some } \quad \boldsymbol{h}^{*} \in \mathcal{A}_{s, k, t} .
$$

Define the function

$$
f(\boldsymbol{x})=c \exp \left(-2 \pi \mathrm{i} \boldsymbol{h}^{*} \cdot \boldsymbol{x}\right) g(\boldsymbol{x})=c \sum_{h \in \mathcal{A}_{s, k, t}} b_{\boldsymbol{h}} \exp \left(2 \pi \mathrm{i}\left(\boldsymbol{h}-\boldsymbol{h}^{*}\right) \cdot \boldsymbol{x}\right),
$$

where a positive $c$ is chosen such that $\|f\| \leq 1$. More precisely, we have

$$
\begin{aligned}
\|f\|^{2} & =c^{2} \sum_{\boldsymbol{h} \in \mathcal{A}_{s, k, t}}\left|b_{\boldsymbol{h}}\right|^{2} \frac{1}{\omega_{\boldsymbol{h}-\boldsymbol{h}^{*}}} \\
& \leq c^{2} \sum_{\boldsymbol{h} \in \mathcal{A}_{s, k, t}} \frac{1}{\omega_{\boldsymbol{h}-\boldsymbol{h}^{*}}} \leq c^{2} \max _{\boldsymbol{h}^{*} \in \mathcal{A}_{s, k, t}} \sum_{\boldsymbol{h} \in \mathcal{A}_{s, k, t}} \frac{1}{\omega_{\boldsymbol{h}-\boldsymbol{h}^{*}}} .
\end{aligned}
$$

Hence, we can take

$$
c=\left[\max _{\boldsymbol{h}^{*} \in \mathcal{A}_{s, k, t}} \sum_{\boldsymbol{h} \in \mathcal{A}_{s, k, t}} \frac{1}{\omega_{\boldsymbol{h}-\boldsymbol{h}^{*}}}\right]^{-1 / 2} .
$$

Note that $f\left(\boldsymbol{t}_{m}\right)=0$ and this implies that $A_{n, s}(f)=0$. Furthermore, $I(f)=$ $c b_{\boldsymbol{h}^{*}}=c$. Hence,

$$
e^{\text {wor }}\left(A_{n, s}\right) \geq\left|I(f)-A_{n, s}(f)\right|=I(f)=c .
$$

Since this holds for all $a_{m}$ and $\boldsymbol{t}_{m}$, we conclude that $e(n, s) \geq c$, as claimed.

\section{LatTice RUles}

In this section we choose our linear algorithms for approximating multivariate integrals as lattice rules of rank one. They are a special case of quasi-Monte Carlo algorithms for which the sample points $t_{m}=\{(m-1) \boldsymbol{g} / n\}$, where $\{\boldsymbol{x}\}$ denotes the fractional parts (componentwisely) of the vector $\boldsymbol{x}$, and $\boldsymbol{g} \in\{0,1, \ldots, n-1\}^{s}$ is called a generator of a lattice rule with $n$ assumed to be prime. Hence, lattice rules are given by

$$
A_{n, s}(f)=\frac{1}{n} \sum_{m=1}^{n} f\left(\left\{\frac{(m-1) \boldsymbol{g}}{n}\right\}\right)
$$

with $\boldsymbol{g} \in\{0,1, \ldots, n-1\}^{s}$ and $n$ is prime. 
4.1. Unweighted case. In this subsection we consider probably the most natural choice of $\omega_{\boldsymbol{h}}$ for which the Korobov space consists of infinitely times differentiable functions. Namely, we take

$$
\omega_{\boldsymbol{h}}=\omega^{|\boldsymbol{h}|} \text { for some } \omega \in(0,1)
$$

Note that for all vectors $\boldsymbol{h} \in \mathbb{Z}^{s}$ for which $|\boldsymbol{h}|=v$ for some positive integer $v$, we have $\omega_{\boldsymbol{h}}=\omega^{v}$. In particular, if we permute components of $\boldsymbol{h}$, then we do not change the coefficient $\omega_{\boldsymbol{h}}$. Hence, if we permute variables of $f \in H(K)$ and obtain the function

$$
g(\boldsymbol{x})=f\left(x_{j_{1}}, x_{j_{2}}, \ldots, x_{j_{s}}\right)
$$

for some permutation $\left(j_{1}, j_{2}, \ldots, j_{s}\right)$ of $(1,2, \ldots, s)$, then $g \in H(K)$ and $\|g\|=\|f\|$. In this sense the choice of $\omega_{\boldsymbol{h}}=\omega^{|\boldsymbol{h}|}$ does not distinguish successive variables and that is why it is called unweighted.

The reproducing kernel now takes the form

$$
K(\boldsymbol{x}, \boldsymbol{y})=\sum_{\boldsymbol{h} \in \mathbb{Z}^{s}} \omega^{|\boldsymbol{h}|} \exp (2 \pi \mathrm{i} \boldsymbol{h} \cdot(\boldsymbol{x}-\boldsymbol{y})) \quad \text { for all } \quad \boldsymbol{x}, \boldsymbol{y} \in[0,1]^{s} .
$$

To stress the role of the generator $\boldsymbol{g}$, we denote the worst-case error of the lattice rule $A_{n, s}$ by $e_{n, s}(\boldsymbol{g}):=e^{\text {wor }}\left(A_{n, s}\right)$. It is known that ([7])

$$
e_{n, s}^{2}(\boldsymbol{g})=\sum_{\boldsymbol{h} \in \mathcal{L}_{\boldsymbol{g}} \backslash\{\mathbf{0}\}} \omega^{|\boldsymbol{h}|},
$$

where the dual lattice is given by

$$
\mathcal{L}_{\boldsymbol{g}}=\left\{\boldsymbol{h} \in \mathbb{Z}^{s}: \boldsymbol{h} \cdot \boldsymbol{g} \equiv 0(\bmod n)\right\} .
$$

We define a suitable figure of merit by

$$
\rho(\boldsymbol{g})=\min _{\boldsymbol{h} \in \mathcal{L}_{\boldsymbol{g}} \backslash\{\mathbf{0}\}}|\boldsymbol{h}| .
$$

Note that this figure of merit is the same as the enhanced trigonometric degree of a lattice rule; see [3, 4, 8, 13].

We bound the worst-case error using the figure of merit in the following lemma.

Lemma 1. Let $n$ be a prime. Then for any $\boldsymbol{g} \in\{0,1, \ldots, n-1\}^{s}$ we have

$$
\omega^{\rho(\boldsymbol{g})} \leq e_{n, s}^{2}(\boldsymbol{g}) \leq \omega^{\rho(\boldsymbol{g})} 2^{s}(1-\omega)^{-s}\left(\begin{array}{c}
\rho(\boldsymbol{g})+s-1 \\
s-1
\end{array}\right) .
$$

Proof. We have

$$
\begin{aligned}
& e_{n, s}^{2}(\boldsymbol{g})=\sum_{\boldsymbol{h} \in \mathcal{L}_{\boldsymbol{g}} \backslash\{\mathbf{0}\}} \omega^{|\boldsymbol{h}|}=\sum_{k=\rho(\boldsymbol{g})}^{\infty} \sum_{\substack{\boldsymbol{h} \in \mathcal{L}_{\boldsymbol{g}} \backslash\{\mathbf{0}\} \\
|\boldsymbol{h}|=k}} \omega^{|\boldsymbol{h}|} \\
& =\sum_{k=\rho(\boldsymbol{g})}^{\infty} \omega^{k} \sum_{\substack{\boldsymbol{h} \in \mathcal{L}_{\boldsymbol{g}} \backslash\{\mathbf{0}\} \\
|\boldsymbol{h}|=k}} 1 \leq \sum_{k=\rho(\boldsymbol{g})}^{\infty} \omega^{k} 2^{s}\left(\begin{array}{c}
k+s-1 \\
s-1
\end{array}\right) \\
& \leq \omega^{\rho(\boldsymbol{g})} 2^{s}(1-\omega)^{-s}\left(\begin{array}{c}
\rho(\boldsymbol{g})+s-1 \\
s-1
\end{array}\right) \text {, }
\end{aligned}
$$


where we used

$$
\sum_{k=\rho}^{\infty}\left(\begin{array}{c}
k+r-1 \\
r-1
\end{array}\right) \omega^{k} \leq \omega^{\rho}\left(\begin{array}{c}
\rho+r-1 \\
r-1
\end{array}\right)(1-\omega)^{-r}
$$

which can be shown using the binomial theorem; see [9, Lemma 2.18] or [5, Lemma $6]$. On the other hand, from the first line above we also have $e_{n, s}^{2}(\boldsymbol{g}) \geq \omega^{\rho(\boldsymbol{g})}$.

We now prove an existence result of generators $\boldsymbol{g}$ with a large figure of merit.

Lemma 2. For a prime number $n$, there exists a $\boldsymbol{g} \in\{0,1, \ldots, n-1\}^{\text {s }}$ such that

$$
\rho(\boldsymbol{g}) \geq\left\lceil 2^{-1}(s ! n)^{1 / s}\right\rceil-s .
$$

Proof. For a given $\boldsymbol{h}=\left(h_{1}, \ldots, h_{s}\right) \in \mathbb{Z}^{s} \backslash\{\mathbf{0}\}$ with $\left|h_{i}\right|<n$ for $1 \leq i \leq s$, there are $n^{s-1}$ choices of $\boldsymbol{g} \in\{0,1, \ldots, n-1\}^{s}$ such that $\boldsymbol{g} \cdot \boldsymbol{h} \equiv 0(\bmod n)$. Furthermore,

$$
\left|\left\{\boldsymbol{h} \in \mathbb{Z}^{s}:|\boldsymbol{h}|=\ell\right\}\right| \leq 2^{s}\left(\begin{array}{c}
\ell+s-1 \\
s-1
\end{array}\right) .
$$

Let $\rho<n$ be a given positive integer (note that $\rho(\boldsymbol{g})<n$ always). Then

$$
\left|\left\{\boldsymbol{h} \in \mathbb{Z}^{s}:|\boldsymbol{h}| \leq \rho\right\}\right| \leq 2^{s} \sum_{\ell=0}^{\rho}\left(\begin{array}{c}
\ell+s-1 \\
s-1
\end{array}\right)=2^{s}\left(\begin{array}{c}
\rho+s \\
s
\end{array}\right) .
$$

Therefore,

$$
\left|\left\{\boldsymbol{g} \in\{0,1, \ldots, n-1\}^{s}: \rho(\boldsymbol{g}) \leq \rho\right\}\right| \leq n^{s-1} 2^{s}\left(\begin{array}{c}
\rho+s \\
s
\end{array}\right) .
$$

Note that the total number of possible generators $\boldsymbol{g} \in\{0,1, \ldots, n-1\}^{s}$ is $n^{s}$. Thus, if

$$
n^{s-1} 2^{s}\left(\begin{array}{c}
\rho+s \\
s
\end{array}\right)<n^{s}
$$

then there exists a $\boldsymbol{g} \in\{0,1, \ldots, n-1\}^{s}$ such that $\rho(\boldsymbol{g})>\rho$. We estimate

$$
2^{s}\left(\begin{array}{c}
\rho+s \\
s
\end{array}\right) \leq 2^{s}(\rho+s)^{s}(s !)^{-1}
$$

Thus (7) is satisfied if $2^{s}(\rho+s)^{s}(s !)^{-1}<n$, i.e., for $\rho=\left\lceil 2^{-1}(s ! n)^{1 / s}\right\rceil-s-1$.

An upper bound on the figure of merit is presented in [8, Section 5].

Lemma 3. For any $n \in \mathbb{N}$ and any $\boldsymbol{g} \in\{0,1, \ldots, n-1\}^{s}$ we have

$$
\rho(\boldsymbol{g}) \leq(s ! n)^{1 / s} \text {. }
$$

Remark 1. It is possible to give in some sense explicit examples of "good lattice points" satisfying

$$
\rho(\boldsymbol{g}) \geq c(s) n^{1 / s}
$$

for some positive $c(s)$. But it seems to be not so easy to obtain $c(s)$ as large as in the existence proof of Lemma 2 .

For example, consider an algebraic number field $F$ of degree $s+1$ and let $1, \delta_{1}, \ldots, \delta_{s}$ be algebraic integers forming a basis of $F$. Let $\delta_{j}^{(1)}, \ldots, \delta_{j}^{(s)}$ be the conjugates of $\delta_{j}, 1 \leq j \leq s$. 
For an integer $n \geq 1$, let $g_{j}=g_{j}(n)$ be the nearest integer to $\delta_{j} n$. Then by Dirichlet's theorem [2, p. 23], for any integer $N \geq 1$ there exists an $n, 1 \leq n \leq N$, such that

$$
\max _{1 \leq j \leq s}\left|\delta_{j}-\frac{g_{j}(n)}{n}\right| \leq \frac{1}{n N^{1 / s}} .
$$

Now let $\boldsymbol{h}=\left(h_{1}, \ldots, h_{s}\right) \in \mathbb{Z}^{s} \backslash\{\mathbf{0}\}$ be such that $g_{1} h_{1}+\cdots+g_{s} h_{s} \equiv 0(\bmod n)$. For $1 \leq j \leq s$ define

$$
x_{j}=h_{1} \delta_{1}^{(j)}+\cdots+h_{s} \delta_{s}^{(j)}-\frac{h_{1} g_{1}+\cdots+h_{s} g_{s}}{n}
$$

and

$$
x_{s+1}=h_{1} \delta_{1}+\cdots+h_{s} \delta_{s}-\frac{h_{1} g_{1}+\cdots+h_{s} g_{s}}{n} .
$$

Then we have

$$
\begin{aligned}
\left|x_{s+1}\right| & =\left|h_{1} \delta_{1}+\cdots+h_{s} \delta_{s}-h_{1} \frac{g_{1}}{n}-\cdots-h_{s} \frac{g_{s}}{n}\right| \\
& \leq \sum_{j=1}^{s}\left|h_{j}\right|\left|\delta_{j}-\frac{g_{j}}{n}\right| \leq \frac{|\boldsymbol{h}|}{n N^{1 / s}} .
\end{aligned}
$$

Furthermore, for $1 \leq j \leq s$, we have

$$
\begin{aligned}
\left|x_{j}\right| & \leq\left|x_{j}-x_{s+1}\right|+\left|x_{s+1}\right| \leq \sum_{i=1}^{s}\left|h_{i}\right|\left|\delta_{i}^{(j)}-\delta_{i}\right|+\frac{|\boldsymbol{h}|}{n N^{1 / s}} \\
& \leq|\boldsymbol{h}|\left(\max _{1 \leq i \leq s}\left|\delta_{i}^{(j)}-\delta_{i}\right|+\frac{1}{n N^{1 / s}}\right) .
\end{aligned}
$$

By the definition of the $x_{j}$, the product $x_{1} \cdots x_{s+1}$ is a nonzero integer, and, therefore,

$$
1 \leq\left|x_{1} \cdots x_{s+1}\right| \leq \frac{|\boldsymbol{h}|^{s+1}}{n N^{1 / s}} \prod_{j=1}^{s}\left(\max _{1 \leq i \leq s}\left|\delta_{i}^{(j)}-\delta_{i}\right|+\frac{1}{n N^{1 / s}}\right) .
$$

Let $\kappa=\prod_{j=1}^{s}\left(\max _{1 \leq i \leq s}\left|\delta_{i}^{(j)}-\delta_{i}\right|+\frac{1}{n N^{1 / s}}\right)$. Then

$$
|\boldsymbol{h}| \geq \frac{n^{1 /(s+1)} N^{1 /(s(s+1))}}{\kappa^{1 /(s+1)}}
$$

and, therefore, for $\boldsymbol{g}(n)=\left(g_{1}(n), \ldots, g_{s}(n)\right)$ we have

$$
\rho(\boldsymbol{g}(n)) \geq \frac{n^{1 /(s+1)} N^{1 /(s(s+1))}}{\kappa^{1 /(s+1)}} .
$$

Using the fact that $1 \leq n \leq N$, the last inequality implies that

$$
\rho(\boldsymbol{g}(n)) \geq \frac{n^{1 / s}}{\kappa^{1 /(s+1)}} .
$$

If, we take, for example, $\delta_{j}=2^{j /(s+1)}, 1 \leq j \leq s$, then $\max _{1 \leq i \leq s}\left|\delta_{i}^{(j)}-\delta_{i}\right| \leq 4$ for all $j$, i.e., $\kappa \leq 5^{s}$. Hence,

$$
\rho(\boldsymbol{g}(n)) \geq \frac{n^{1 / s}}{5}
$$


Note that the generating vectors $\boldsymbol{g}=\boldsymbol{g}(n)$ can easily be computed. By calculating $\boldsymbol{g}=\boldsymbol{g}(n)$ for $n=1,2, \ldots$ and calculating $\rho(\boldsymbol{g}(n))$ to check the figure of merit, one must find infinitely many "good" generating vectors for which

$$
\frac{\rho(\boldsymbol{g}(n))}{n^{1 / s}} \geq \kappa^{-1 /(s+1)} .
$$

Indeed, inequality (8) is effective in obtaining an upper bound on the number of points needed to increase the figure of merit by one in the following way: Assume that for some $n^{*}>1$ we found a generating vector $\boldsymbol{g}^{*}=\boldsymbol{g}\left(n^{*}\right)$ with figure of merit $\rho^{*}=\rho\left(\boldsymbol{g}^{*}\right)$ such that $\rho(\boldsymbol{g}(n))<\rho^{*}$ for all $1 \leq n<n^{*}$. Then, from (8), we know that there exists an $n$ with $n^{*}<n \leq N^{*}$ and a vector $\boldsymbol{g}(n)$ with figure of merit $\rho(\boldsymbol{g}(n))=\rho^{*}+1$, where

$$
N^{*}=\left\lfloor\left(\frac{\kappa\left(\rho^{*}\right)^{s+1}}{n^{*}+1}\right)^{s}\right\rfloor+1 .
$$

Thus, $N^{*}-n^{*}$ is the maximum waiting period till the next increase of the figure of merit must occur. In particular, this shows that there exists a sequence of integers $n_{1}, n_{2}, \ldots$ with $1 \leq n_{1}<n_{2}<n_{3}<\cdots, \rho\left(\boldsymbol{g}\left(n_{1}\right)\right)<\rho\left(\boldsymbol{g}\left(n_{2}\right)\right)<\rho\left(\boldsymbol{g}\left(n_{3}\right)\right)<\cdots$, and $\rho\left(\boldsymbol{g}\left(n_{k}\right)\right) \geq n_{k}^{1 / s} \kappa^{-1 /(s+1)}$ for $k=1,2,3, \ldots$

This search method may also be interesting in the context of finding lattice rules with a moderately large trigonometric degree for parameters $s$ and $n$, where an (or nearly) exhaustive computer search for good lattice rules cannot be undertaken.

Combining Lemmas 1, 2 and 3, we obtain the following theorem.

Theorem 2. Let $e_{n, s}(\boldsymbol{g})$ denote the worst-case error of the lattice rule with generator $\boldsymbol{g}$ and with $n$ points in dimension $s$.

- For a prime number $n$, there exists a generator $\boldsymbol{g} \in\{0,1, \ldots, n-1\}^{s}$ such that

$$
e_{n, s}^{2}(\boldsymbol{g}) \leq \omega^{2^{-1}(s ! n)^{1 / s}}\left(\frac{4 \mathrm{e}}{\omega-\omega^{2}}\right)^{s} n
$$

- For any $n \in \mathbb{N}$ and any $\boldsymbol{g} \in\{0,1, \ldots, n-1\}^{s}$ we have

$$
e_{n, s}^{2}(\boldsymbol{g}) \geq \omega^{(s ! n)^{1 / s}} \text {. }
$$

Proof. From Lemma 10 we have

$$
\begin{aligned}
e_{n, s}^{2}(\boldsymbol{g}) & \leq \omega^{\rho(\boldsymbol{g})} 2^{s}(1-\omega)^{-s}\left(\begin{array}{c}
\rho(\boldsymbol{g})+s-1 \\
s-1
\end{array}\right) \\
& \leq \omega^{\rho(\boldsymbol{g})} 2^{s}(1-\omega)^{-s} \frac{(\rho(\boldsymbol{g})+s-1)^{s-1}}{(s-1) !} .
\end{aligned}
$$

From Lemma 2 we know that there exists a generator $\boldsymbol{g} \in\{0,1, \ldots, n-1\}^{s}$ with

$$
\rho(\boldsymbol{g}) \geq 2^{-1}(s ! n)^{1 / s}-s,
$$


and from Lemma 3 we know that $\rho(\boldsymbol{g}) \leq(s ! n)^{1 / s}$. Inserting these estimates into (91), we have

$$
\begin{aligned}
e_{n, s}^{2}(\boldsymbol{g}) & \leq \omega^{2^{-1}(s ! n)^{1 / s}-s} 2^{s}(1-\omega)^{-s} \frac{\left((s ! n)^{1 / s}+s-1\right)^{s-1}}{(s-1) !} \\
& \leq \omega^{2^{-1}(s ! n)^{1 / s}} 2^{s}\left(\omega-\omega^{2}\right)^{-s} \frac{s^{s-1}\left(n^{1 / s}+1\right)^{s-1}}{(s-1) !} \\
& \leq \omega^{2^{-1}(s ! n)^{1 / s}}(2 \mathrm{e})^{s}\left(\omega-\omega^{2}\right)^{-s}\left(n^{1 / s}+1\right)^{s-1} \\
& \leq \omega^{2^{-1}(s ! n)^{1 / s}}(4 \mathrm{e})^{s}\left(\omega-\omega^{2}\right)^{-s} n .
\end{aligned}
$$

This proves the first estimate.

The second estimate easily follows from Lemmas 1 and 3 .

It is natural to ask how good are the error bounds presented in Theorem 2 for lattice rules. First of all, it is easy to see that the upper bound on $e_{n, s}(\boldsymbol{g})$ converges faster than any power of $n$ as $n$ goes to infinity. That is, for arbitrarily large $r$ we have

$$
\lim _{n \text { prime, } n \rightarrow \infty} \frac{e_{n, s}(\boldsymbol{g})}{n^{r}}=0 \text { for all } s \in \mathbb{N} .
$$

Indeed, $e_{n, s}(\boldsymbol{g}) / n^{r} \leq x_{n}$, where

$$
2 \ln x_{n}=\frac{1}{2}(s ! n)^{1 / s} \ln \omega+s \ln \left(4 \mathrm{e} /\left(\omega-\omega^{2}\right)\right)+\ln n-r \ln n \rightarrow-\infty,
$$

so that $x_{n}$ goes to zero, as claimed.

Does it mean that we have exponential convergence? Assume for a moment that the dimension $s$ cannot go to infinity, say, $s \in\left[1, s^{*}\right]$ for an arbitrary integer $s^{*}$. Then there exists a positive $C$ such that

$$
e_{n, s}(\boldsymbol{g}) \leq C \omega^{n^{1 /\left(1+s^{*}\right)}} \quad \text { for all primes } n \text { and } s \in\left[1, s^{*}\right] .
$$

Indeed, since $(s !)^{1 / s} \geq s / \mathrm{e}$, and this inequality is asymptotically sharp due to Stirling's formula, we have

$$
\begin{aligned}
& \sup _{n \text { primes, } s \in\left[1, s^{*}\right]} \frac{e_{n, s}(\boldsymbol{g})}{\omega^{n^{1 /\left(1+s^{*}\right)}}} \\
& \leq C:=\left(\frac{4 \mathrm{e}}{\omega-\omega^{2}}\right)^{s^{*} / 2} \sup _{n \in \mathbb{N}, s \in\left[1, s^{*}\right]} \sqrt{n} \omega^{s /(4 \mathrm{e}) n^{1 / s}-n^{1 /\left(1+s^{*}\right)}}<\infty .
\end{aligned}
$$

This means exponential convergence for a restricted range of $s$. In this case, we want to find a prime $n$ for which $e_{n, s} \leq \varepsilon$. It is enough to find an integer $n$ that is not necessarily prime for which $C \omega^{n^{1 /\left(1+s^{*}\right)}} \leq \varepsilon$, and then use the fact that we can find a prime in the interval $[n, 2 n]$. This yields the bound

$$
n(\varepsilon, s) \leq 2\left\lceil\left(\frac{\ln C+\ln \varepsilon^{-1}}{\ln \omega^{-1}}\right)^{1+s^{*}}\right\rceil \text { for all } s \in\left[1, s^{*}\right], \varepsilon \in(0,1) .
$$

Hence, we obtain exponential convergence with strong polynomial tractability for a restricted range of $s$. However, we stress that the exponent of $\ln \varepsilon^{-1}$ is $1+s^{*}$ and for large $s^{*}$ it can be quite harmful.

Consider now the case when $s$ can go to infinity. Then the lower bound on $e_{n, s}(\boldsymbol{g})$ in Theorem 2 implies that exponential convergence does not hold. Indeed, 
for any $q \in(0,1)$ and $p>0$, we estimate $(s !)^{1 / s} \leq s$, take $s>1 / p$ so that $p>1 / s$, and then

$$
\limsup _{n \rightarrow \infty} \frac{e_{n, s}(\boldsymbol{g})}{q^{n^{p}}} \geq \limsup _{n \rightarrow \infty} \exp \left(-\frac{1}{2} s n^{1 / s} \ln \omega^{-1}+n^{p} \ln q^{-1}\right)=\infty .
$$

This means that we cannot achieve exponential convergence as long as we use lattice rules. But maybe it is possible to achieve exponential convergence and at least weak tractability if we use different algorithms. Unfortunately, it is not the case as shown in the next theorem.

Theorem 3. Exponential convergence with weak tractability of multivariate integration for the Korobov space with $\omega_{\boldsymbol{h}}=\omega^{|\boldsymbol{h}|}$, where $\omega \in(0,1)$, does not hold.

Proof. We use Theorem 1. For all $\boldsymbol{h} \in \mathcal{A}_{s, k, t}$, we have $\omega_{\boldsymbol{h}} \geq \omega^{t k}$. Furthermore, note that $\boldsymbol{h}-\boldsymbol{h}^{*}$ has at most $2 k$ nonzero components and these nonzero components are from $\{-t,-t+1, \ldots, t\}$. Therefore,

$$
\omega_{\boldsymbol{h}-\boldsymbol{h}^{*}} \geq \omega^{2 t k} \quad \text { for all } \boldsymbol{h}, \boldsymbol{h}^{*} \in \mathcal{A}_{s, k, t} .
$$

Hence,

$$
\max _{\boldsymbol{h}^{*} \in \mathcal{A}_{s, k, t}} \sum_{\boldsymbol{h} \in \mathcal{A}_{s, k, t}} \frac{1}{\omega_{\boldsymbol{h}-\boldsymbol{h}^{*}}} \leq \frac{\left|\mathcal{A}_{s, k, t}\right|}{\omega^{2 t k}}=\left(\begin{array}{l}
s \\
k
\end{array}\right) \frac{t^{k}}{\omega^{2 t k}} .
$$

Theorem 1 yields that

$$
e^{2}(n, s) \geq \frac{\omega^{2 t k}}{\left(\begin{array}{l}
s \\
k
\end{array}\right) t^{k}} \quad \text { for all } n<\left(\begin{array}{l}
s \\
k
\end{array}\right) t^{k} .
$$

Suppose that we have exponential convergence and weak tractability. Then

$$
e(n, s) \leq C(s) q^{n^{p}} \text { for all } s, n \in \mathbb{N},
$$

with $\ln C(s)=\exp (o(s))$ as well as $q \in(0,1)$ and $p>0$.

Now take $t=s$ and $k=\lfloor s / 2\rfloor$ with

$$
n=\left(\begin{array}{l}
s \\
k
\end{array}\right) t^{k}-1=\Theta\left(\frac{2^{s} s^{\lfloor s / 2\rfloor}}{s^{1 / 2}}\right) \text {. }
$$

Then for large $s$, we have $n>s^{s / 2}$ and

$$
1 \leq\left(\begin{array}{l}
s \\
k
\end{array}\right)^{1 / 2} t^{k / 2} \omega^{-t k} C(s) q^{s p / 2} .
$$

Taking the logarithms we conclude that

$$
0 \leq \frac{1}{2}(1+o(1)) s^{2} \ln \omega^{-1}+\exp (o(s))-s^{s p / 2} \ln q^{-1} .
$$

For large $s$, the last inequality is not true since the right-hand side tends to $-\infty$. This completes the proof.

In summary, the choice of $\omega_{\boldsymbol{h}}=\omega^{|\boldsymbol{h}|}$ yields exponential convergence with strong polynomial tractability for a restricted range of $s$, and this can be achieved by using lattice rules. We think it is quite a positive result as long as the range of $s$ is restricted to $\left[1, s^{*}\right]$ with a relatively small $s^{*}$. However, if we allow $s$ to be arbitrarily large, the choice of $\omega_{\boldsymbol{h}}=\omega^{|\boldsymbol{h}|}$ does not yield exponential convergence and weak tractability. We need to consider smaller coefficients $\omega_{\boldsymbol{h}}$ to achieve our goal of exponential convergence and at least weak tractability. 
4.2. Weighted case. In this section we consider the Korobov space for which successive variables and groups of variables may play a different role. This is achieved by introducing three sequences of nonnegative weights

$$
\gamma=\left\{\gamma_{s, \mathfrak{u}}\right\}_{s \in \mathbb{N}, \mathfrak{u} \subseteq[s]}, \quad c=\left\{c_{s, \mathfrak{u}}\right\}_{s \in \mathbb{N}, \mathfrak{u} \subseteq[s]}, \quad \beta=\left\{\beta_{s, \mathfrak{u}}\right\}_{s \in \mathbb{N}, \mathfrak{u} \subseteq[s]},
$$

where

$$
[s]:=\{1,2, \ldots, s\}
$$

If $s$ is clear from the context, we will be using the shorthand notation $\gamma_{\mathfrak{u}}=\gamma_{s, \mathfrak{u}}$, $c_{\mathfrak{u}}=c_{s, \mathfrak{u}}$ and $\beta_{\mathfrak{u}}=\beta_{s, \mathfrak{u}}$. We always assume that $\gamma_{\emptyset}=1$ and $c_{\emptyset}=0$ as well as that $\beta_{\mathfrak{u}} \geq 1$ and $c_{\mathfrak{u}} \geq c_{0}>0$ for all nonempty $\mathfrak{u} \subseteq[s]$.

For $\boldsymbol{h} \in \mathbb{Z}^{s}$ we denote $\mathfrak{u}_{\boldsymbol{h}}=\left\{j \in[s]: h_{j} \neq 0\right\}$. We choose the coefficients

$$
\omega_{\boldsymbol{h}}=\gamma_{\mathfrak{u}_{\boldsymbol{h}}} \omega^{c_{\mathfrak{u}_{\boldsymbol{h}}}|\boldsymbol{h}|^{\beta_{\mathfrak{u}_{\boldsymbol{h}}}}} \quad \text { for all } \boldsymbol{h} \in \mathbb{Z}^{s}
$$

where $\omega \in(0,1)$. Note that $\omega_{0}=1$ and

$$
\sum_{\boldsymbol{h} \in \mathbb{Z}^{s}} \omega_{\boldsymbol{h}} \leq \max _{\mathfrak{u} \subseteq[s]} \gamma_{\mathfrak{u}} \sum_{\boldsymbol{h} \in \mathbb{Z}^{s}} \omega^{c_{0}|\boldsymbol{h}|}=\max _{\mathfrak{u} \subseteq[s]} \gamma_{\mathfrak{u}}\left(1+\frac{2}{1-\omega^{c_{0}}}\right)^{s}<\infty
$$

as needed in (11).

This corresponds to the reproducing kernel given by

$$
K(\boldsymbol{x}, \boldsymbol{y})=\sum_{\mathfrak{u} \subseteq[s]} \gamma_{\mathfrak{u}} \sum_{\boldsymbol{h}_{\mathfrak{u}} \in(\mathbb{Z} \backslash\{0\})^{|\mathfrak{u}|}} \omega^{c_{\mathfrak{u}}\left|\boldsymbol{h}_{\mathfrak{u}}\right|^{\beta_{\mathfrak{u}}}} \exp \left(2 \pi i\left(\boldsymbol{h}_{\mathfrak{u}}, \mathbf{0}\right) \cdot(\boldsymbol{x}-\boldsymbol{y})\right)
$$

for all $\boldsymbol{x}, \boldsymbol{y} \in[0,1]^{s}$. Here, for $\boldsymbol{h}_{\mathfrak{u}} \in(\mathbb{Z} \backslash\{0\})^{|\mathfrak{u}|}$, the $j$ th component of the vector $\left(\boldsymbol{h}_{\mathfrak{u}}, \mathbf{0}\right) \in \mathbb{Z}^{s}$ is $h_{j}$ if $j \in \mathfrak{u}$ and 0 if $j \notin \mathfrak{u}$.

The worst-case error $e_{n, s}(\boldsymbol{g})$ of the lattice rule with generator $\boldsymbol{g} \in\{0,1, \ldots, n-$ $1\}^{s}$ is now

$$
e_{n, s}^{2}(\boldsymbol{g})=\sum_{\emptyset \neq \mathfrak{u} \subseteq[s]} \gamma_{\mathfrak{u}} \sum_{\boldsymbol{h}_{\mathfrak{u}} \in \mathcal{L}_{\mathfrak{u}, \boldsymbol{g}}} \omega^{c_{\mathfrak{u}}\left|\boldsymbol{h}_{\mathfrak{u}}\right|^{\beta_{\mathfrak{u}}}}
$$

where the dual lattice is given by

$$
\mathcal{L}_{\mathfrak{u}, \boldsymbol{g}}=\left\{\boldsymbol{h}_{\mathfrak{u}} \in(\mathbb{Z} \backslash\{0\})^{|\mathfrak{u}|}:\left(\boldsymbol{h}_{\mathfrak{u}}, \mathbf{0}\right) \cdot \boldsymbol{g} \equiv 0(\bmod n)\right\} .
$$

We define a suitable figure of merit by

$$
\rho_{\mathfrak{u}}(\boldsymbol{g})=\min _{\boldsymbol{h}_{\mathfrak{u}} \in \mathcal{L}_{\mathfrak{u}, g}}\left|\boldsymbol{h}_{\mathfrak{u}}\right|
$$

Note that $\rho_{\mathfrak{u}}(\boldsymbol{g}) \geq|\mathfrak{u}|$. Furthermore, we set

$$
\rho(\boldsymbol{g})=\min _{\emptyset \neq \mathfrak{u} \subseteq[s]} \rho_{\mathfrak{u}}(\boldsymbol{g}) .
$$

Lemma 4. For $0<\omega<1$ and integers $\rho \geq r \geq 1$ and $\sigma \geq 1$, we have

$$
\sum_{k=\rho}^{\infty}\left(\begin{array}{l}
k-1 \\
r-1
\end{array}\right) \omega^{k^{\sigma}} \leq \omega^{\rho^{\sigma}}\left(\begin{array}{l}
\rho-1 \\
r-1
\end{array}\right)(1-\omega)^{-r}
$$


Proof. We transform

$$
\begin{aligned}
\sum_{k=\rho}^{\infty}\left(\begin{array}{l}
k-1 \\
r-1
\end{array}\right) \omega^{k^{\sigma}} & =\omega^{\rho^{\sigma}} \sum_{k=\rho}^{\infty}\left(\begin{array}{c}
k-1 \\
r-1
\end{array}\right) \omega^{k^{\sigma}-\rho^{\sigma}} \leq \omega^{\rho^{\sigma}} \sum_{k=\rho}^{\infty}\left(\begin{array}{l}
k-1 \\
r-1
\end{array}\right) \omega^{k-\rho} \\
& =\omega^{\rho^{\sigma}-\rho} \sum_{k=\rho}^{\infty}\left(\begin{array}{c}
k-r+r-1 \\
r-1
\end{array}\right) \omega^{k} \\
& =\omega^{\rho^{\sigma}-\rho+r} \sum_{k=\rho-r}^{\infty}\left(\begin{array}{c}
k+r-1 \\
r-1
\end{array}\right) \omega^{k} .
\end{aligned}
$$

Using (6) we conclude

$$
\sum_{k=\rho}^{\infty}\left(\begin{array}{l}
k-1 \\
r-1
\end{array}\right) \omega^{k^{\sigma}} \leq \omega^{\rho^{\sigma}-\rho+r} \omega^{\rho-r}\left(\begin{array}{l}
\rho-1 \\
r-1
\end{array}\right)(1-\omega)^{-r} .
$$

We bound the worst-case error using the figure of merit in the following lemma.

Lemma 5. The worst-case error $e_{n, s}(\boldsymbol{g})$ of the lattice rule with generator $\boldsymbol{g}$ and with $n$ points in dimension $s$ is bounded by

$$
\sum_{\emptyset \neq \mathfrak{u} \subseteq[s]} \gamma_{\mathfrak{u}} \omega^{c_{\mathfrak{u}} \rho_{\mathfrak{u}}(\boldsymbol{g})^{\beta_{\mathfrak{u}}}} \leq e_{n, s}^{2}(\boldsymbol{g}) \leq \sum_{\emptyset \neq \mathfrak{u} \subseteq[s]} \omega^{c_{\mathfrak{u}} \rho_{\mathfrak{u}}(\boldsymbol{g})^{\beta_{\mathfrak{u}}}} \frac{\left.\gamma_{\mathfrak{u}}\right|^{|\mathfrak{u}|}}{\left(1-\omega^{c_{\mathfrak{u}}}\right)^{|\mathfrak{u}|}}\left(\begin{array}{c}
\rho_{\mathfrak{u}}(\boldsymbol{g})-1 \\
|\mathfrak{u}|-1
\end{array}\right) .
$$

Proof. We have

$$
\begin{aligned}
& e_{n, s}^{2}(\boldsymbol{g})=\sum_{\emptyset \neq \mathfrak{u} \subseteq[s]} \gamma_{\mathfrak{u}} \sum_{\boldsymbol{h}_{\mathfrak{u}} \in \mathcal{L}_{\mathfrak{u}, \boldsymbol{g}}} \omega^{c_{\mathfrak{u}} \mid \boldsymbol{h}_{\mathfrak{u}} \beta^{\beta_{\mathfrak{u}}}} \\
& =\sum_{\emptyset \neq \mathfrak{u} \subseteq[s]} \gamma_{\mathfrak{u}} \sum_{k=\rho_{\mathfrak{u}}(\boldsymbol{g})}^{\infty} \sum_{\substack{\boldsymbol{h}_{\mathfrak{u}} \in \mathcal{L}_{\mathfrak{u}, g} \\
\left|\boldsymbol{h}_{\mathfrak{u}}\right|=k}} \omega^{c_{\mathfrak{u}}\left|\boldsymbol{h}_{\mathfrak{u}}\right|^{\beta_{\mathfrak{u}}}} \\
& =\sum_{\emptyset \neq u \in[s]} \gamma_{\mathfrak{u}} \sum_{k=\rho_{\mathfrak{u}}(\boldsymbol{g})}^{\infty} \omega^{c_{\mathfrak{u}} k^{\beta_{\mathfrak{u}}}} \sum_{\substack{\boldsymbol{h}_{\mathfrak{u}} \in \mathcal{L}_{\mathfrak{u}, g} \\
\left|\boldsymbol{h}_{\mathfrak{u}}\right|=k}} 1 \\
& \leq \sum_{\emptyset \neq \mathfrak{u} \subseteq[s]} \gamma_{\mathfrak{u}} \sum_{k=\rho_{\mathfrak{u}}(\boldsymbol{g})}^{\infty} \omega^{c_{\mathfrak{u}} k^{\beta_{\mathfrak{u}}}} 2^{|\mathfrak{u}|}\left(\begin{array}{c}
k-1 \\
|\mathfrak{u}|-1
\end{array}\right) \\
& \leq \sum_{\emptyset \neq \mathfrak{u} \subseteq[s]} \omega^{c_{\mathfrak{u}} \rho_{\mathfrak{u}}(\boldsymbol{g})^{\beta_{\mathfrak{u}}}} \frac{\gamma_{\mathfrak{u}} 2^{|\mathfrak{u}|}}{\left(1-\omega^{c_{\mathfrak{u}}}\right)^{|\mathfrak{u}|}}\left(\begin{array}{c}
\rho_{\mathfrak{u}}(\boldsymbol{g})-1 \\
|\mathfrak{u}|-1
\end{array}\right),
\end{aligned}
$$

which is the upper bound. To prove the lower bound, we use (10)

$$
e_{n, s}(\boldsymbol{g})=\sum_{\emptyset \neq \mathfrak{u} \subseteq[s]} \gamma_{\mathfrak{u}} \sum_{k=\rho_{\mathfrak{u}}(\boldsymbol{g})}^{\infty} \omega^{c_{\mathfrak{u}} k^{\beta_{\mathfrak{u}}}} \sum_{\substack{\boldsymbol{h}_{\mathfrak{u}} \in \mathcal{L}_{\mathfrak{u}, g} \\\left|\boldsymbol{h}_{\mathfrak{u}}\right|=k}} 1 \geq \sum_{\emptyset \neq \mathfrak{u} \subseteq[s]} \gamma_{\mathfrak{u}} \omega^{c_{\mathfrak{u}} \rho_{\mathfrak{u}}(\boldsymbol{g})^{\beta_{\mathfrak{u}}}} .
$$

Next we prove an existence result for generators $\boldsymbol{g}$ with a large figure of merit.

Lemma 6. For a prime number $n$ and arbitrary positive real numbers $d_{\mathfrak{u}}$ with

$$
\sum_{\emptyset \neq \mathfrak{u} \subseteq[s]} d_{\mathfrak{u}} \leq 1
$$


there exists a generator $\boldsymbol{g} \in\{0,1, \ldots, n-1\}^{s}$ such that

$$
\rho_{\mathfrak{u}}(\boldsymbol{g}) \geq\left\lceil 2^{-1}\left(|\mathfrak{u}| ! d_{\mathfrak{u}} n\right)^{1 /|\mathfrak{u}|}\right\rceil-1 \quad \text { for all nonempty } \mathfrak{u} \subseteq[s] .
$$

Proof. For each given $\boldsymbol{h} \in \mathbb{Z}^{s} \backslash\{\mathbf{0}\}$ there are $n^{s-1}$ choices of $\boldsymbol{g} \in\{0,1, \ldots, n-1\}^{s}$ such that $\boldsymbol{g} \cdot \boldsymbol{h} \equiv 0(\bmod n)$. For $\emptyset \neq \mathfrak{u} \subseteq[s]$ and for $\ell \geq|\mathfrak{u}|$, we have

$$
\left|\left\{\boldsymbol{h}_{\mathfrak{u}} \in(\mathbb{Z} \backslash\{0\})^{|\mathfrak{u}|}:\left|\boldsymbol{h}_{\mathfrak{u}}\right|=\ell\right\}\right|=2^{|\mathfrak{u}|}\left(\begin{array}{c}
\ell-1 \\
|\mathfrak{u}|-1
\end{array}\right) .
$$

Take an integer $\rho_{\mathfrak{u}}$ such that $\rho_{\mathfrak{u}} \geq|\mathfrak{u}|$. Then

$$
\left|\left\{\boldsymbol{h}_{\mathfrak{u}} \in(\mathbb{Z} \backslash\{0\})^{|\mathfrak{u}|}:\left|\boldsymbol{h}_{\mathfrak{u}}\right| \leq \rho_{\mathfrak{u}}\right\}\right|=2^{|\mathfrak{u}|} \sum_{\ell=|\mathfrak{u}|}^{\rho_{\mathfrak{u}}}\left(\begin{array}{c}
\ell-1 \\
|\mathfrak{u}|-1
\end{array}\right)=2^{|\mathfrak{u}|}\left(\begin{array}{c}
\rho_{\mathfrak{u}} \\
|\mathfrak{u}|
\end{array}\right) .
$$

Therefore,

$$
\left|\left\{\boldsymbol{g} \in\{0,1, \ldots, n-1\}^{s}: \rho_{\mathfrak{u}}(\boldsymbol{g}) \leq \rho_{\mathfrak{u}}\right\}\right| \leq n^{s-1} 2^{|\mathfrak{u}|}\left(\begin{array}{c}
\rho_{\mathfrak{u}} \\
|\mathfrak{u}|
\end{array}\right)
$$

Thus, if

$$
n^{s-1} 2^{|\mathfrak{u}|}\left(\begin{array}{l}
\rho_{\mathfrak{u}} \\
|\mathfrak{u}|
\end{array}\right)<d_{\mathfrak{u}} n^{s}
$$

where $n^{s}$ is the total number of possible generators, there exist more than $\left(1-d_{\mathfrak{u}}\right) n^{s}$ generators $\boldsymbol{g} \in\{0,1, \ldots, n-1\}^{s}$ such that $\rho_{\mathfrak{u}}(\boldsymbol{g})>\rho_{u}$. We have

$$
2^{|\mathfrak{u}|}\left(\begin{array}{c}
\rho_{\mathfrak{u}} \\
|\mathfrak{u}|
\end{array}\right) \leq \frac{2^{|\mathfrak{u}|}}{|\mathfrak{u}| !} \rho_{\mathfrak{u}}^{|\mathfrak{u}|}
$$

Thus (11) is satisfied for $\rho_{\mathfrak{u}}$ for which $2^{|\mathfrak{u}|}(|\mathfrak{u}| !)^{-1} \rho_{\mathfrak{u}}^{|\mathfrak{u}|}<d_{\mathfrak{u}} n$, that is, for

$$
\rho_{\mathfrak{u}}=\left\lceil 2^{-1}\left(|\mathfrak{u}| ! d_{\mathfrak{u}} n\right)^{1 /|\mathfrak{u}|}\right\rceil-1 \text {. }
$$

For $\emptyset \neq \mathfrak{u} \subseteq[s]$, let

$$
A_{\mathfrak{u}}=\left\{\boldsymbol{g} \in\{0,1, \ldots, n-1\}^{s}: \rho_{\mathfrak{u}}(\boldsymbol{g})>\rho_{\mathfrak{u}}\right\}
$$

and $A=\bigcap_{\emptyset \neq \mathfrak{u} \subseteq[s]} A_{\mathfrak{u}}$. Let $A^{\prime}=\{0,1, \ldots, n-1\}^{s} \backslash A$, similarly define $A_{\mathfrak{u}}^{\prime}$. Then we have

$$
\left|A^{\prime}\right|=\left|\bigcup_{\emptyset \neq \mathfrak{u} \subseteq[s]} A_{\mathfrak{u}}^{\prime}\right| \leq \sum_{\emptyset \neq \mathfrak{u} \subseteq[s]}\left|A_{\mathfrak{u}}^{\prime}\right|<n^{s} \sum_{\emptyset \neq \mathfrak{u} \subseteq[s]} d_{\mathfrak{u}} \leq n^{s} .
$$

Thus, the set $A$ is nonempty, and there exists a $\boldsymbol{g} \in\{0,1, \ldots, n-1\}^{s}$ such that $\rho_{\mathfrak{u}}(\boldsymbol{g})>\rho_{\mathfrak{u}}$ for all $\emptyset \neq \mathfrak{u} \subseteq[s]$, as claimed.

Lemma 7. For $\omega_{1} \in(\omega, 1)$ and $c_{0}>0$, there exists a positive number $C=$ $C\left(\omega, \omega_{1}, c\right)$ such that

$$
\left(\begin{array}{l}
x-1 \\
k-1
\end{array}\right) \omega^{c x^{k}} \leq C \omega_{1}^{c x^{k}} \quad \text { for all } \quad x, k \in \mathbb{N} \text { and } c \geq c_{0} .
$$

Proof. Let $q:=\omega / \omega_{1}$. Clearly, $q \in(0,1)$. Then

$$
\begin{aligned}
\left(\begin{array}{l}
x-1 \\
k-1
\end{array}\right) q^{c x^{k}} & \leq(x-1)^{k-1} q^{c x^{k}}<x^{k} q^{c x^{k}} \leq x^{k} q^{c_{0} x^{k}} \\
& \leq \sup _{m \in \mathbb{N}} m q^{c_{0} m}=: C<\infty,
\end{aligned}
$$

as claimed. 
Combining Lemmas 5, 6 and 7, we obtain the following theorem.

Theorem 4. Assume that

$$
\beta_{\mathfrak{u}} \geq|\mathfrak{u}| \quad \text { and } \quad c_{\mathfrak{u}} \geq c_{0}>0 \quad \text { for all nonempty } \mathfrak{u} \subseteq[s] .
$$

Let $\omega_{1} \in(\omega, 1)$. For a prime number $n$ and arbitrary positive real numbers $d_{\mathfrak{u}}=$ $d_{s, \mathfrak{u}}$,

$$
\emptyset \neq \mathfrak{u} \subseteq[s], \quad \text { with } \sum_{\emptyset \neq \mathfrak{u} \subseteq[s]} d_{\mathfrak{u}} \leq 1,
$$

there exists a generator $\boldsymbol{g} \in\{0,1, \ldots, n-1\}^{s}$ such that

$$
e_{n, s}^{2}(\boldsymbol{g}) \leq C_{1} \sum_{\emptyset \neq \mathfrak{u} \subseteq[s]} \omega_{1}^{c_{\mathfrak{u}} 4^{-|\mathfrak{u}|}|\mathfrak{u}| ! d_{\mathfrak{u}} n} \gamma_{\mathfrak{u}} 2^{|\mathfrak{u}|}\left(1-\omega^{c_{\mathfrak{u}}}\right)^{-|\mathfrak{u}|}
$$

where the positive constant $C_{1}$ depends only on $\omega, \omega_{1}$ and $c_{0}$.

Proof. From Lemma 5, the assumption on the $\beta_{\mathfrak{u}}$ 's and Lemma 7 we obtain

$$
\begin{aligned}
e_{n, s}^{2}(\boldsymbol{g}) & \leq \sum_{\emptyset \neq \mathfrak{u} \subseteq[s]} \omega^{c_{\mathfrak{u}} \rho_{\mathfrak{u}}(\boldsymbol{g})^{\beta_{\mathfrak{u}}}} \gamma_{\mathfrak{u}} 2^{|\mathfrak{u}|}\left(1-\omega^{c_{\mathfrak{u}}}\right)^{-|\mathfrak{u}|}\left(\begin{array}{c}
\rho_{\mathfrak{u}}(\boldsymbol{g})-1 \\
|\mathfrak{u}|-1
\end{array}\right) \\
& \leq \sum_{\emptyset \neq \mathfrak{u} \subseteq[s]} \omega^{c_{\mathfrak{u}} \rho_{\mathfrak{u}}(\boldsymbol{g})^{|\mathfrak{u}|}} \gamma_{\mathfrak{u}} 2^{|\mathfrak{u}|}\left(1-\omega^{c_{\mathfrak{u}}}\right)^{-|\mathfrak{u}|}\left(\begin{array}{c}
\rho_{\mathfrak{u}}(\boldsymbol{g})-1 \\
|\mathfrak{u}|-1
\end{array}\right) \\
& \leq\left. C_{1} \sum_{\emptyset \neq \mathfrak{u} \subseteq[s]} \omega_{1}^{c_{\mathfrak{u}} \rho_{\mathfrak{u}}(\boldsymbol{g})^{|\mathfrak{u}|}} \gamma_{\mathfrak{u}}\right|^{|\mathfrak{u}|}\left(1-\omega^{c_{\mathfrak{u}}}\right)^{-|\mathfrak{u}|} .
\end{aligned}
$$

Using Lemma 6 and the fact that $\rho_{\mathfrak{u}}(\boldsymbol{g}) \geq|\mathfrak{u}| \geq 1$ for any nonempty $\mathfrak{u}$, there exists a generator $\boldsymbol{g} \in\{0,1, \ldots, n-1\}^{s}$ for which

$$
e_{n, s}^{2}(\boldsymbol{g}) \leq C_{1} \sum_{\emptyset \neq \mathfrak{u} \subseteq[s]} \omega_{1}^{c_{\mathfrak{u}}\left(\max \left\{\left\lceil 2^{-1}\left(|\mathfrak{u}| ! d_{\mathfrak{u}} n\right)^{1 /|\mathfrak{u}|}\right\rceil-1,1\right\}\right)^{|\mathfrak{u}|}} \gamma_{\mathfrak{u}} 2^{|\mathfrak{u}|}\left(1-\omega^{c_{\mathfrak{u}}}\right)^{-|\mathfrak{u}|} .
$$

If $2^{-1}\left(|\mathfrak{u}| ! d_{\mathfrak{u}} n\right)^{1 /|\mathfrak{u}|}>1$, then we have

$$
\left\lceil 2^{-1}\left(|\mathfrak{u}| ! d_{\mathfrak{u}} n\right)^{1 /|\mathfrak{u}|}\right\rceil-1 \geq 4^{-1}\left(|\mathfrak{u}| ! d_{\mathfrak{u}} n\right)^{1 /|\mathfrak{u}|} .
$$

If $2^{-1}\left(|\mathfrak{u}| ! d_{\mathfrak{u}} n\right)^{1 /|\mathfrak{u}|} \leq 1$, then we have

$$
\max \left\{\left\lceil 2^{-1}\left(|\mathfrak{u}| ! d_{\mathfrak{u}} n\right)^{1 /|\mathfrak{u}|}\right\rceil-1,1\right\}=1 \geq 2^{-1}\left(|\mathfrak{u}| ! d_{\mathfrak{u}} n\right)^{1 /|\mathfrak{u}|} .
$$

Hence, in both cases, we have

$$
\max \left\{\left\lceil 2^{-1}\left(|\mathfrak{u}| ! d_{\mathfrak{u}} n\right)^{1 /|\mathfrak{u}|}\right\rceil-1,1\right\} \geq 4^{-1}\left(|\mathfrak{u}| ! d_{\mathfrak{u}} n\right)^{1 /|\mathfrak{u}|}
$$

and, therefore,

$$
e_{n, s}^{2}(\boldsymbol{g}) \leq C_{1} \sum_{\emptyset \neq \mathfrak{u} \subseteq[s]} \omega_{1}^{c_{\mathfrak{u}} 4^{-|\mathfrak{u}|}|\mathfrak{u}| ! d_{\mathfrak{u}} n} \gamma_{\mathfrak{u}} 2^{|\mathfrak{u}|}\left(1-\omega^{c_{\mathfrak{u}}}\right)^{-|\mathfrak{u}|},
$$

as claimed. 


\section{Tractability}

We present conditions on the weights $\beta_{s, \mathfrak{u}}, c_{s, \mathfrak{u}}$ and $\gamma_{s, \mathfrak{u}}$ to obtain tractability and exponential convergence. We recall that these weights define $\omega_{s, \boldsymbol{h}}$ by

$$
\omega_{s, \boldsymbol{h}}=\gamma_{s, \mathfrak{u}_{\boldsymbol{h}}} \omega^{c_{s, u_{h}}}|\boldsymbol{h}|^{\beta_{s, u_{h}}} \quad \text { for all } \boldsymbol{h} \in \mathbb{Z}^{s}
$$

with $\mathfrak{u}_{\boldsymbol{h}}=\left\{j \in[s]: h_{j} \neq 0\right\}$.

Theorem 5. Choose $\beta_{s, \mathfrak{u}}$ and $c_{s, \mathfrak{u}}$ such that

$$
\beta_{s, \mathfrak{u}} \geq|\mathfrak{u}| \quad \text { and } \quad c_{s, \mathfrak{u}} \geq c_{0}>0
$$

for all $s \in \mathbb{N}$ and all nonempty $\mathfrak{u} \subseteq[s]$. Assume that

$$
\limsup _{s \rightarrow \infty} \sum_{\emptyset \neq \mathfrak{u} \subseteq[s]} \frac{4^{|\mathfrak{u}|}}{c_{s, \mathfrak{u}}|\mathfrak{u}| !}<\infty .
$$

Let $\omega_{1} \in(\omega, 1)$. Then for every prime $n$ and any dimension $s \in \mathbb{N}$ there exists a generator $\boldsymbol{g} \in\{0,1, \ldots, n-1\}^{s}$ such that

$$
e_{n, s}^{2}(\boldsymbol{g}) \leq C_{1} C(s) \omega_{1}^{c n},
$$

where a positive number $C_{1}$ depends only on $\omega, \omega_{1}$ and $c_{0}$,

$$
C(s)=\sum_{\emptyset \neq \mathfrak{u} \subseteq[s]} \gamma_{s, \mathfrak{u}} 2^{|\mathfrak{u}|}\left(1-\omega^{c_{s, \mathfrak{u}}}\right)^{-|\mathfrak{u}|}
$$

and

$$
\frac{1}{c}=\sup _{s \in \mathbb{N}} \sum_{\emptyset \neq \mathfrak{u} \subseteq[s]} \frac{4^{|\mathfrak{u}|}}{c_{s, \mathfrak{u}}|\mathfrak{u}| !}<\infty
$$

In particular,

- if

$$
\sup _{s \in \mathbb{N}} C(s)<\infty
$$

then we have exponential convergence with strong polynomial tractability, and

$$
n(\varepsilon, s)=\mathcal{O}\left(1+\ln \varepsilon^{-1}\right) \quad \text { for all } \quad \varepsilon \in(0,1), s \in \mathbb{N},
$$

- if there exists a positive $\tau$ such that

$$
\sup _{s \in \mathbb{N}} s^{-\tau} \ln (1+C(s))<\infty
$$

then we have exponential convergence with polynomial tractability, and

$$
n(\varepsilon, s)=\mathcal{O}\left(s^{\tau}+\ln \varepsilon^{-1}\right) \quad \text { for all } \varepsilon \in(0,1), s \in \mathbb{N},
$$

- if

$$
\lim _{s \rightarrow \infty} \frac{\ln (1+\ln (1+C(s)))}{s}=0,
$$

then we have exponential convergence and weak tractability, and

$$
n(\varepsilon, s)=\mathcal{O}\left(\exp (o(s))+\ln \varepsilon^{-1}\right) \quad \text { for all } \varepsilon \in(0,1), s \in \mathbb{N} .
$$

In all three cases, the factor in the big $\mathcal{O}$ notation is independent of $\varepsilon^{-1}$ and $s$. 
Proof. First of all, note that $c>0$. Indeed, we assumed that the limit superior of

$$
\sum_{\emptyset \neq \mathfrak{u} \subseteq[s]} 4^{|\mathfrak{u}|} /\left(c_{s, \mathfrak{u}}|\mathfrak{u}| !\right)
$$

is finite, and therefore the supremum over $s$ of the same sum is finite. Hence, $1 / c<\infty$ and $c>0$.

For any $\emptyset \neq \mathfrak{u} \subseteq[s]$, define

$$
d_{s, \mathfrak{u}}=\frac{c 4^{|\mathfrak{u}|}}{c_{s, \mathfrak{u}}|\mathfrak{u}| !} .
$$

Then $\sum_{\emptyset \neq \mathfrak{u} \subseteq[s]} d_{s, \mathfrak{u}} \leq 1$. By Theorem 4 there exists a generator $\boldsymbol{g} \in\{0,1, \ldots, n-$ $1\}^{s}$ such that

$$
\begin{aligned}
& e_{n, s}^{2}(\boldsymbol{g}) \leq C_{1} \sum_{\emptyset \neq \mathfrak{u} \subseteq[s]} \omega_{1}^{c_{s, \mathfrak{u}} 4^{-|\mathfrak{u}|}|\mathfrak{u}| ! d_{s, \mathfrak{u}} n} \gamma_{s, \mathfrak{u}} 2^{|\mathfrak{u}|}\left(1-\omega^{c_{s, \mathfrak{u}}}\right)^{-|\mathfrak{u}|} \\
& =C_{1} \omega_{1}^{c n} \sum_{\emptyset \neq \mathfrak{u} \subseteq[s]} \gamma_{s, \mathfrak{u}} 2^{|\mathfrak{u}|}\left(1-\omega^{c_{s, \mathfrak{u}}}\right)^{-|\mathfrak{u}|} \\
& =C_{1} C(s) \omega_{1}^{c n} \text {, }
\end{aligned}
$$

where $C_{1}>0$ is as in Theorem 4 , and hence depends only on $\omega, \omega_{1}$ and $c_{0}$.

This means that we have exponential convergence. The conditions on tractability in terms of $C(s)$ have already been established in Section 2. This completes the proof.

We now show how to find $c$ and $c_{0}$ for

$$
c_{s, \mathfrak{u}}=(|\mathfrak{u}| !)^{-1} 4^{|\mathfrak{u}|} \prod_{j \in \mathfrak{u}} j^{\alpha} \quad \text { with } \quad \alpha>1
$$

for all nonempty $\mathfrak{u} \subseteq[s]$. Then

$$
\begin{aligned}
\sum_{\emptyset \neq \mathfrak{u} \subseteq[s]} \frac{4^{|\mathfrak{u}|}}{c_{s, \mathfrak{u}}|\mathfrak{u}| !} & =\sum_{\emptyset \neq \mathfrak{u} \subseteq[s]} \prod_{j \in \mathfrak{u}} j^{-\alpha} \\
& =\sum_{k=1}^{s} \sum_{\mathfrak{u} \subseteq \mathbb{N},|\mathfrak{u}|=k} \prod_{j \in \mathfrak{u}} j^{-\alpha} \\
& \leq \sum_{k=1}^{\infty}(k !)^{-1} \zeta(\alpha)^{k}=\exp (\zeta(\alpha))-1,
\end{aligned}
$$

where $\zeta(\alpha)=\sum_{j=1}^{\infty} j^{-\alpha}$ is the Riemann zeta function. Therefore, $c \geq(\exp (\zeta(\alpha))-$ $1)^{-1}$. Since

$$
c_{s, \mathfrak{u}}=(|\mathfrak{u}| !)^{-1} 4^{|\mathfrak{u}|} \prod_{j \in \mathfrak{u}} j^{\alpha} \geq(|\mathfrak{u}| !)^{\alpha-1} 4^{|\mathfrak{u}|} \geq 4
$$

we can take $c_{0}=4$.

Another choice of $c_{s, \mathfrak{u}}$ is $c_{s, \mathfrak{u}}=c(s)$ for some function $c$. Then we have

$$
\begin{aligned}
\sum_{\emptyset \neq \mathfrak{u} \subseteq[s]} \frac{4^{|\mathfrak{u}|}}{c_{s, \mathfrak{u}}|\mathfrak{u}| !} & =\frac{1}{c(s)} \sum_{k=1}^{s} \frac{4^{k}}{k !}\left(\begin{array}{l}
s \\
k
\end{array}\right) \leq \frac{1}{c(s)} \sum_{k=1}^{s} \frac{4^{k} s^{k}}{k ! k !} \\
& \leq \frac{1}{c(s)} \max _{k \in \mathbb{N}} \frac{4^{k}}{k !} \exp (s)=\frac{32 \exp (s)}{3 c(s)} .
\end{aligned}
$$


Hence, the last sum is uniformly bounded in $s$, for instance, if we take $c(s)=\exp (s)$. For such $c(s)$ we have $c_{0}=\mathrm{e}$ and $c \geq 3 / 32$.

We now illustrate Theorem 5 for product weights. That is, $\gamma_{s, \emptyset}=1$ and

$$
\gamma_{s, \mathfrak{u}}=\prod_{j \in \mathfrak{u}} \gamma_{s, j}
$$

for all nonempty $\mathfrak{u} \subseteq[s]$. Here, $\left\{\gamma_{s, j}\right\}_{s \in \mathbb{N}, j=1,2, \ldots, s}$ is a given sequence of nonnegative numbers. From Theorem 5 we easily obtain the following corollary.

Corollary 1. Consider product weights $\gamma_{s, \mathfrak{u}}$ with $\beta_{s, \mathfrak{u}}$ and $c_{s, \mathfrak{u}}$ satisfying the assumptions of Theorem [5. Then

- if

$$
\limsup _{s \rightarrow \infty} \sum_{j=1}^{s} \gamma_{s, j}<\infty
$$

then we have exponential convergence with strong polynomial tractability, and

$$
n(\varepsilon, s)=\mathcal{O}\left(1+\ln \varepsilon^{-1}\right) \quad \text { for all } \varepsilon \in(0,1), s \in \mathbb{N},
$$

- if there exists a positive $\tau$ such that

$$
\limsup _{s \rightarrow \infty} s^{-\tau} \sum_{j=1}^{s} \gamma_{s, j}<\infty,
$$

then we have exponential convergence with polynomial tractability, and for any positive $\delta$ we have

$$
n(\varepsilon, s)=\mathcal{O}\left(s^{\tau+\delta}+\ln \varepsilon^{-1}\right) \quad \text { for all } \varepsilon \in(0,1), s \in \mathbb{N},
$$

- if

$$
\lim _{s \rightarrow \infty} \frac{\ln \sum_{j=1}^{s} \gamma_{s, j}}{s}=0,
$$

then we have exponential convergence with weak tractability, and

$$
n(\varepsilon, s)=\mathcal{O}\left(\exp (o(s))+\ln \varepsilon^{-1}\right) \quad \text { for all } \varepsilon \in(0,1), s \in \mathbb{N} .
$$

In all three cases, the factor in the big $\mathcal{O}$ notation is independent of $\varepsilon^{-1}$ and $s$.

Proof. We have

$$
C(s)=\sum_{\emptyset \neq \mathfrak{u} \subseteq[s]} \gamma_{s, \mathfrak{u}} 2^{|\mathfrak{u}|}\left(1-\omega^{c_{s, \mathfrak{u}}}\right)^{-|\mathfrak{u}|} .
$$

Since $c_{s, \mathfrak{u}} \geq c_{0}>0$ for all nonempty $\mathfrak{u} \subseteq[s]$, we obtain

$$
\begin{aligned}
C(s) & \leq \sum_{\emptyset \neq \mathfrak{u} \subseteq[s]} \prod_{j \in \mathfrak{u}} \gamma_{s, j} \frac{2}{1-\omega^{c_{0}}}=\prod_{j=1}^{s}\left(1+\gamma_{s, j} \frac{2}{1-\omega^{c_{0}}}\right)-1 \\
& \leq \exp \left(\ln \left(\prod_{j=1}^{s}\left(1+\gamma_{s, j} \frac{2}{1-\omega^{c_{0}}}\right)\right)\right) \\
& =\exp \left(\sum_{j=1}^{s} \ln \left(1+\gamma_{s, j} \frac{2}{1-\omega^{c_{0}}}\right)\right) \leq \exp \left(\frac{2}{1-\omega^{c_{0}}} \sum_{j=1}^{s} \gamma_{s, j}\right) .
\end{aligned}
$$


Hence

$$
\ln (1+C(s))=\mathcal{O}\left(1+\sum_{j=1}^{s} \gamma_{s, j}\right) .
$$

The rest easily follows from Theorem 5 and the assumptions on $\sum_{j=1}^{s} \gamma_{s, j}$.

Note that for $\gamma_{s, j}=1$ the condition on strong polynomial tractability is not satisfied, but we have polynomial tractability with $\tau=1$. For $\gamma_{s, j}=s^{k}$, polynomial tractability still holds, however, now $\tau=k+1$. Finally, for $\gamma_{s, j}=j^{s^{a}}$, the condition on polynomial tractability does not hold for any positive $a$, however, weak tractability holds for any $a<1$.

Remark 2. We now discuss the role of the weights $\beta_{s, \mathfrak{u}}$. They are more important than the weights $\gamma_{s, \mathfrak{u}}$ and $c_{s, \mathfrak{u}}$ since they determine the powers of $|\boldsymbol{h}|$ in the exponents of $\omega$. In Theorem 5 we assumed that $\beta_{s, \mathfrak{u}} \geq|\mathfrak{u}|$. Obviously, it is possible to modify Theorem [5 with the assumption $\beta_{s, \mathfrak{u}} \geq \beta_{0}|\mathfrak{u}|$ for some positive $\beta_{0}$. We choose $\beta_{0}=1$ to simplify the notation. However, the choice $\beta_{s, \mathfrak{u}}=o(|\mathfrak{u}|)$ with the same assumptions on $\gamma_{s, \mathfrak{u}}$ and $c_{s, \mathfrak{u}}$ contradicts exponential convergence with weak tractability as we will now show. From this point of view the choice $\beta_{s, \mathfrak{u}}=\Omega(|\mathfrak{u}|)$ is best possible.

For simplicity, take $\gamma_{s, \mathfrak{u}}=1$ and $c_{s, \mathfrak{u}}=\exp (s)$. We know that this and $\beta_{s, \mathfrak{u}} \geq|\mathfrak{u}|$ yield exponential convergence with polynomial tractability.

We now assume that $\beta_{s, \mathfrak{u}}=\beta(|\mathfrak{u}|)=o(|\mathfrak{u}|)$ for some monotonically increasing function $\beta$ such that $\beta(|\mathfrak{u}|) \geq 1$. We show that exponential convergence with weak tractability no longer holds. To prove this we use Theorem 1 and proceed similarly as in Theorem 3. By the conditions on the weights, for all $\boldsymbol{h}, \boldsymbol{h}^{*} \in \mathcal{A}_{s, k, t}$ we have

$$
\omega_{\boldsymbol{h}-\boldsymbol{h}^{*}} \geq \omega^{\exp (s)(2 t k)^{\beta(2 k)}} .
$$

Hence,

$$
e^{2}(n, s) \geq \frac{\omega^{\exp (s)(2 t k)^{\beta(2 k)}}}{\left(\begin{array}{l}
s \\
k
\end{array}\right) t^{k}}
$$

for all $n<\left(\begin{array}{l}s \\ k\end{array}\right) t^{k}$.

Without loss of generality, we assume that $s$ is even and take $t=s, k=s / 2$ and $n=\left(\begin{array}{l}s \\ k\end{array}\right) t^{k}-1=\Theta\left(\frac{2^{s} s^{s / 2}}{s^{1 / 2}}\right)$.

Suppose that exponential convergence with weak tractability holds. Then there exist a positive $p$ and $q \in(0,1)$ such that

$$
e(n, s) \leq \exp (\exp (o(s))) q^{n^{p}} \quad \text { for all } \quad s, n \in \mathbb{N} .
$$

This leads to

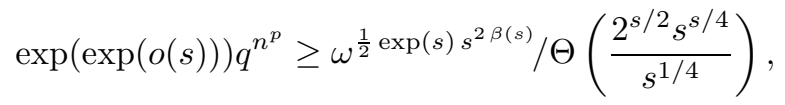

and taking the logarithms we obtain

$$
\begin{array}{r}
\exp (o(s))+\Theta\left(\frac{2^{s p} s^{s p / 2}}{s^{p / 2}}\right) \log q-\frac{\exp (s) s^{2 o(s)} \log \omega}{2} \\
+\Theta\left(\frac{s \log 2}{2}+\frac{(s-1) \log s}{4}\right) \geq 0 .
\end{array}
$$


For large $s$, this reduces to

$$
\exp (s) s^{o(s)} \geq \Theta\left(2^{s p} s^{(s-1) p / 2}\right)
$$

which is a contradiction.

Remark 3 . We stress that we can obtain strong polynomial tractability with weaker assumptions on the weights if we only demand a polynomial convergence instead of the exponential one. In this case, we can even choose $\beta_{s, \mathfrak{u}}=c_{s, \mathfrak{u}}=1$. Then

$$
\omega_{s, \mathfrak{u}_{h}}=\gamma_{s, \mathfrak{u}_{h}} \omega^{|h|} \text { for all } \boldsymbol{h} \in \mathbb{Z}^{s} .
$$

By the usual averaging argument using Jensen's inequality (see for example [18]), we obtain that for any $s \in \mathbb{N}$ and any prime $n$ there exists a $\boldsymbol{g} \in\{0,1, \ldots, n-1\}^{s}$ such that

$$
e_{n, s}^{2}(\boldsymbol{g}) \leq \frac{1}{(n-1)^{\alpha}}\left(\sum_{\emptyset \neq \mathfrak{u} \subseteq[s]} \gamma_{s, \mathfrak{u}}^{1 / \alpha}\left(\frac{2 \omega^{1 / \alpha}}{1-\omega^{1 / \alpha}}\right)^{|\mathfrak{u}|}\right)^{\alpha},
$$

for positive $\alpha$ that can be arbitrarily large. If

$$
C_{\alpha}:=\sup _{s \in \mathbb{N}} \sum_{\emptyset \neq \mathfrak{u} \subseteq[s]} \gamma_{s, \mathfrak{u}}^{1 / \alpha}\left(\frac{2 \omega^{1 / \alpha}}{1-\omega^{1 / \alpha}}\right)^{|\mathfrak{u}|}<\infty,
$$

then we have

$$
n(\varepsilon, s) \leq\left\lceil C_{\alpha} \varepsilon^{-2 / \alpha}\right\rceil,
$$

which means strong polynomial tractability. If the weights $\gamma_{s, \mathfrak{u}}$ are of product form independent of $s$, i.e.,

$$
\gamma_{s, \mathfrak{u}}=\prod_{j \in \mathfrak{u}} \gamma_{j}
$$

where $\left\{\gamma_{j}\right\}_{j \in \mathbb{N}}$ is a sequence of nonnegative reals, then condition (13) is satisfied iff

$$
\sum_{j=1}^{\infty} \gamma_{j}^{1 / \alpha}<\infty
$$

\section{Constructive approach}

We present a constructive result, now in the weighted setting. The "pseudoconstructive" point set of Remark 1 no longer works. However, it seems natural to use sample points from regular grids with different mesh-sizes that depend on the weights. In the following we assume that we are given an increasing sequence

$$
1 \leq \beta(1) \leq \beta(2) \leq \ldots
$$

of positive reals such that $\beta^{*}:=\sum_{i=1}^{\infty} 1 / \beta(i)<\infty$.

For $s, m \in \mathbb{N}$, let the point set $\mathcal{P}_{s}$ be given by

$$
\left(\left\{\frac{k_{1}}{m_{1}}\right\}, \ldots,\left\{\frac{k_{s}}{m_{s}}\right\}\right)
$$

for $k_{i}=0,1, \ldots, m_{i}-1$ and $i=1,2, \ldots, s$, where $m_{i}:=\left\lfloor m^{1 /\left(\beta^{*} \cdot \beta(i)\right)}\right\rfloor$.

The cardinality of the point set $\mathcal{P}_{s}$ is

$$
n=\prod_{i=1}^{s}\left\lfloor m^{1 /\left(\beta^{*} \cdot \beta(i)\right)}\right\rfloor \leq m^{\frac{1}{\beta^{*}} \sum_{i=1}^{s} \beta(i)^{-1}} \leq m .
$$


The point set $\mathcal{P}_{s}$ is a grid with the mesh-size $1 / m_{i}$ that does not decrease with the coordinate direction $i$. The mesh-size is small for the important directions that correspond to small weight $\beta(i)$, and becomes larger and larger for less important directions corresponding to large weights $\beta(i)$. In particular, since $\beta^{*} \cdot \beta(i) \geq i$ and goes to infinity with $i$, we have $m_{i}=1$ for large $i$.

Theorem 6. Choose the following weights:

- $\gamma_{s, \mathfrak{u}}=1 \quad$ for all $s \in \mathbb{N}$ and $\mathfrak{u} \subseteq[s]$,

- $\beta_{s, \mathfrak{u}}=\beta\left(\max _{j \in \mathfrak{u}} j\right)$ for all $s \in \mathbb{N}$ and nonempty $\mathfrak{u} \subseteq[s]$, where $\beta: \mathbb{N} \rightarrow \mathbb{N}$ is some function such that

$$
1 \leq \beta(1) \leq \beta(2) \leq \ldots \quad \text { and } \quad \beta^{*}:=\sum_{i=1}^{\infty} 1 / \beta(i)<\infty .
$$

- $c_{s, \mathfrak{u}}=2^{\beta_{s, u}} \quad$ for all $s \in \mathbb{N}$ and nonempty $\mathfrak{u} \subseteq[s]$.

Then for any $s \in \mathbb{N}$ for the point set $\mathcal{P}_{s}$ defined by (14) with $n=\left|\mathcal{P}_{s}\right|$, we have

$$
e_{n, s}^{2}\left(\mathcal{P}_{s}\right) \leq c^{s} \omega^{n^{1 / \beta^{*}}}
$$

for some $c \geq 1$.

That is, we have exponential convergence with polynomial tractability, and

$$
n(\varepsilon, s)=\mathcal{O}\left(\left(s+\ln \varepsilon^{-1}\right)^{\beta^{*}}\right)
$$

with the factor in the big $\mathcal{O}$ notation independent of $\varepsilon^{-1}$ and $s$.

Proof. The worst-case error for integration using a quasi-Monte Carlo rule with quadrature points $\mathcal{P}_{s}$ and $n=\left|\mathcal{P}_{s}\right|$, is given by

$$
e_{n, s}^{2}\left(\mathcal{P}_{s}\right)=-1+\frac{1}{n^{2}} \sum_{\boldsymbol{x}, \boldsymbol{y} \in \mathcal{P}_{s}} K(\boldsymbol{x}, \boldsymbol{y}) .
$$

Then we have

$$
\begin{aligned}
& e_{n, s}^{2}\left(\mathcal{P}_{s}\right) \\
& =-1+\frac{1}{n^{2}} \sum_{k_{1}, l_{1}=0}^{m_{1}-1} \ldots \sum_{k_{s}, l_{s}=0}^{m_{s}-1} \sum_{\mathfrak{u} \subseteq[s]} \\
& \sum_{\boldsymbol{h}_{\mathfrak{u}} \in(\mathbb{Z} \backslash\{0\})^{|\mathfrak{u}|}} \omega^{c_{\mathfrak{u}}\left|\boldsymbol{h}_{\mathfrak{u}}\right|^{\beta_{\mathfrak{u}}}} \exp \left(2 \pi i \sum_{j \in \mathfrak{u}} \frac{h_{j}\left(k_{j}-l_{j}\right)}{m}\right) \\
& =-1+\sum_{\mathfrak{u} \subseteq[s]} \sum_{\boldsymbol{h}_{\mathfrak{u}} \in(\mathbb{Z} \backslash\{0\})^{|\mathfrak{u}|}} \omega^{c_{\mathfrak{u}}\left|\boldsymbol{h}_{\mathfrak{u}}\right|^{\beta_{\mathfrak{u}}}} \frac{\prod_{j \notin \mathfrak{u}} m_{j}^{2}}{\prod_{i=1}^{s} m_{i}^{2}} \\
& \times \prod_{j \in u} \sum_{k_{j}, l_{j}=0}^{m_{j}-1} \exp \left(2 \pi i h_{j}\left(k_{j}-l_{j}\right) / m_{j}\right) \\
& =-1+\sum_{\mathfrak{u} \subseteq[s]} \sum_{\boldsymbol{h}_{\mathfrak{u}} \in(\mathbb{Z} \backslash\{0\})^{|\mathfrak{u}|}} \omega^{c_{\mathfrak{u}}\left|\boldsymbol{h}_{\mathfrak{u}}\right|^{\beta_{\mathfrak{u}}}} \frac{\prod_{j \notin \mathfrak{u}} m_{j}^{2}}{\prod_{i=1}^{s} m_{i}^{2}} \\
& \times \prod_{j \in u}\left|\sum_{k_{j}=0}^{m_{j}-1} \exp \left(2 \pi i h_{j} k_{j} / m_{j}\right)\right|^{2} \text {. }
\end{aligned}
$$


For any $m \in \mathbb{N}$ and $h \in \mathbb{Z}$ we have

$$
\sum_{k=0}^{m-1} \exp (2 \pi \mathrm{i} h k / m)= \begin{cases}m & \text { if } h \equiv 0(\bmod m), \\ 0 & \text { if } h \neq \equiv(\bmod m) .\end{cases}
$$

Therefore, we obtain

$$
\begin{aligned}
e_{n, s}^{2}\left(\mathcal{P}_{s}\right) & =-1+\sum_{\mathfrak{u} \subseteq[s]} \sum_{\substack{\boldsymbol{h}_{\mathfrak{u}} \in(\mathbb{Z} \backslash\{0\})^{|\mathfrak{u}|} \\
h_{j} \equiv 0\left(\bmod m_{j}\right) \forall j \in \mathfrak{u}}} \omega^{c_{\mathfrak{u}}\left|\boldsymbol{h}_{\mathfrak{u}}\right|^{\beta_{\mathfrak{u}}}} \\
& =-1+\sum_{\mathfrak{u} \subseteq[s]} \sum_{\boldsymbol{a} \in(\mathbb{Z} \backslash\{0\})^{|\mathfrak{u}|}} \omega^{c_{\mathfrak{u}}\left(\left|a_{1} m_{\nu_{1}}\right|+\cdots+\left|a_{\mathfrak{u}} m_{\nu_{\mid \mathfrak{u}} \mid}\right|\right)^{\beta_{\mathfrak{u}}}} \\
& =\sum_{\emptyset \neq \mathfrak{u} \subseteq[s]} \sum_{\boldsymbol{a} \in(\mathbb{Z} \backslash\{0\})^{|\mathfrak{u}|}} \omega^{\left.\left(2\left(\left|a_{1} m_{\nu_{1}}\right|+\cdots+\left|a_{|\mathfrak{u}|} m_{\nu_{|\mathfrak{u}|} \mid}\right|\right)\right)^{\beta(\nu|\mathfrak{u}|}\right)},
\end{aligned}
$$

where $\mathfrak{u}=\left\{\nu_{1}, \cdots, \nu_{|\mathfrak{u}|}\right\}$, with $\nu_{1} \leq \nu_{2} \leq \cdots \leq \nu_{|\mathfrak{u}|}$ and where $\boldsymbol{a}=\left(a_{1}, \ldots, a_{|\mathfrak{u}|}\right)$.

Since $\tau=\beta\left(\nu_{|\mathfrak{u}|}\right) \geq 1$ and $a_{i} \geq 1$, we have

$$
\sum_{i=1}^{|\mathfrak{u}|}\left|a_{i}\right|\left(2 m_{\nu_{i}}\right)^{\tau} \leq \sum_{i=1}^{|\mathfrak{u}|}\left|a_{i}\right|^{\tau}\left(2 m_{\nu_{i}}\right)^{\tau}
$$

By Jensen's inequality, we have

$$
\sum_{i=1}^{|\mathfrak{u}|}\left|a_{i}\right|^{\tau}\left(2 m_{\nu_{i}}\right)^{\tau} \leq\left(\sum_{i=1}^{|\mathfrak{u}|}\left|a_{i}\right| 2 m_{\nu_{i}}\right)^{\tau}
$$

Therefore,

$$
\left(\sum_{i=1}^{|\mathfrak{u}|}\left|a_{i}\right| 2 m_{\nu_{i}}\right)^{\tau} \geq \sum_{i=1}^{|\mathfrak{u}|}\left|a_{i}\right|\left(2 m_{\nu_{i}}\right)^{\tau}
$$

Hence,

$$
\begin{aligned}
& \omega^{\left(2\left(\left|a_{1} m_{\nu_{1}}\right|+\cdots+\left|a_{|\mathfrak{u}|} m_{\nu_{\mid \mathfrak{u}} \mid}\right|\right)\right)^{\beta(\nu|\mathfrak{u}|)}} \leq \omega^{\left(2 m_{\nu_{1}}\right)^{\beta(\nu|\mathfrak{u}|)}\left|a_{1}\right|+\cdots+\left(2 m_{\nu_{\mid \mathfrak{u}} \mid}\right)^{\beta\left(\nu_{|\mathfrak{u}|}\right)}\left|a_{|\mathfrak{u}|}\right|} \\
& \leq \omega^{\frac{\beta(\nu|\mathfrak{u}|)}{\beta \cdot \beta\left(\nu_{1}\right)}}\left|a_{1}\right|+\cdots+m^{\frac{\beta\left(\nu_{|u|}\right)}{\beta \cdot \beta\left(\nu_{|u|} \mid\right)}}\left|a_{|\mathfrak{u}|}\right| \\
& \leq \omega^{\left|a_{1}\right|+\cdots+\left|a_{|\mathfrak{u}|-1}\right|+m^{1 / \beta}\left|a_{|\mathfrak{u}|}\right|} \text {. }
\end{aligned}
$$

Therefore, we have

$$
\sum_{\boldsymbol{a} \in(\mathbb{Z} \backslash\{0\})^{|\mathfrak{u}|}} \omega^{\left(2\left(\left|a_{1} m_{\nu_{1}}\right|+\cdots+\left|a_{|\mathfrak{u}|} m_{\nu_{|\mathfrak{u}|}}\right|\right)\right)^{\beta(\nu|\mathfrak{u}|)}} \leq\left(2 \sum_{a=1}^{\infty} \omega^{a}\right)^{|\mathfrak{u}|-1} 2 \sum_{a=1}^{\infty} \omega^{a m^{1 / \beta^{*}}} .
$$

Hence, for the worst-case error we get

$$
\begin{aligned}
e_{n, s}^{2}\left(\mathcal{P}_{s}\right) & \leq \sum_{\emptyset \neq \mathfrak{u} \subseteq[s]}\left(\frac{2 \omega}{1-\omega}\right)^{|\mathfrak{u}|-1} \frac{2}{1-\omega} \omega^{m^{1 / \beta^{*}}} \\
& \leq \frac{1}{\omega}\left(\frac{2 \omega}{1-\omega}+1\right)^{s} \omega^{m^{1 / \beta^{*}}}
\end{aligned}
$$

and the result follows. 
Remark 4. If we take $\beta$ as in Theorem [6, and redefine

$$
c_{s, \mathfrak{u}}=\frac{\log 3}{\log \omega^{-1}} 2^{\beta_{s, \mathfrak{u}}}
$$

and $\gamma_{s, \mathfrak{u}}$ such that

$$
\sum_{\emptyset \neq \mathfrak{u} \subseteq[s]} \gamma_{s, \mathfrak{u}}<\infty
$$

then following the proof of Theorem 6 it is easy to see that we even obtain the exponential convergence with strong polynomial tractability.

Remark 5. The (essential) weights $\beta_{s, \mathfrak{u}}$ in Theorem [6 are larger than the weights $\beta_{s, \mathfrak{u}}$ in Theorem [5. However, the weights $\beta_{s, \mathfrak{u}}$ in Theorem [6 are again, in some sense, best possible if regular grids with arbitrary mesh-sizes are used. To see this, again take $\gamma_{s, \mathfrak{u}}=1$ and $c_{s, \mathfrak{u}}=2^{\beta_{s, \mathfrak{u}}}$ as in Theorem [6, and assume now that $\beta_{\mathfrak{u}}=\beta\left(\max _{j \in \mathfrak{u}} j\right)$ with a monotonically increasing function $\beta$ such that

$$
\sum_{i=1}^{\infty} 1 / \beta(i)=+\infty
$$

This holds, for instance, for $\beta(i)=i$.

Let $\sigma(s):=\sum_{i=1}^{s} 1 / \beta(i)$. Then for given integers $m_{1}, \ldots, m_{s}, n=m_{1} \cdots m_{s}$, take the grid $\mathcal{P}_{s}$ given by

$$
\left(\left\{\frac{k_{1}}{m_{1}}\right\}, \ldots,\left\{\frac{k_{s}}{m_{s}}\right\}\right)
$$

for $k_{i}=0,1, \ldots, m_{i}-1$ and $i=1,2, \ldots, s$.

From the proof of Theorem 6 we have

$$
\begin{aligned}
& e_{n, s}^{s}\left(\mathcal{P}_{s}\right) \geq \sum_{\emptyset \neq \mathfrak{u} \subseteq[s]} \sum_{\boldsymbol{a} \in(\mathbb{Z} \backslash\{0\})^{|\mathfrak{u}|}} \omega^{\left.\left(2\left(\left|a_{1} m_{\nu_{1}}\right|+\cdots+\left|a_{|\mathfrak{u}|} m_{\nu_{\mid \mathfrak{u}} \mid}\right|\right)\right)^{\beta(\nu|\mathfrak{u}|}\right)} \\
& \geq \sum_{\substack{\nu=1 \\
\mathfrak{u}=\{\nu\}}}^{s} \omega^{2 m_{\nu}^{\beta(\nu)}} \geq \omega^{2 \min _{1 \leq \nu \leq s} m_{\nu}^{\beta(\nu)}} .
\end{aligned}
$$

Even for real $\bar{m}_{\nu}$ with $\bar{m}_{1} \cdots \bar{m}_{s}=n$ we have

$$
\min _{1 \leq \nu \leq s} \bar{m}_{\nu}^{\beta(\nu)} \leq n^{1 / \sigma(s)}
$$

Hence,

$$
e_{n, s}^{2}\left(\mathcal{P}_{s}\right) \geq \omega^{2 n^{1 / \sigma(s)}}
$$

and since $\lim _{s \rightarrow \infty} 1 / \sigma(s)=0$ we cannot have exponential convergence.

\section{REFERENCES}

[1] N. Aronszajn, Theory of reproducing kernels. Trans. Amer. Math. Soc. 68 (1950), 337-404. MR0051437 (14:479c)

[2] Y. Bugeaud, Approximation by Algebraic Numbers, Cambridge University Press, Cambridge, 2004. MR2136100 (2006d:11085)

[3] R. Cools and H. Govaert, Five- and six-dimensional lattice rules generated by structured matrices. Information-Based Complexity Workshop (Minneapolis, MN, 2002). J. Complexity 19 (2003), 715-729. MR2039626 (2005a:65021)

[4] R. Cools and J. N. Lyness, Three- and four-dimensional $K$-optimal lattice rules of moderate trigonometric degree. Math. Comp. 70 (2001), 1549-1567. MR1836918(2002b:41026) 
[5] J. Dick and F. Pillichshammer, On the mean square weighted $\mathcal{L}_{2}$ discrepancy of randomized digital $(t, m, s)$-nets over $\mathbb{Z}_{2}$. Acta Arith. 117 (2005), 371-403. MR2140164 (2005k:11152)

[6] J. Dick, F. Pillichshammer, and B. J. Waterhouse, The construction of good extensible rank-1 lattices. Math. Comp. 77 (2008), 2345-2373. MR2429889 (2009f:11095)

[7] F. J. Hickernell, Lattice rules: How well do they measure up? In: Random and quasi-random point sets, P. Hellekalek and G. Larcher (eds.), pp. 109-166, Lecture Notes in Statist., 138, Springer, New York, 1998. MR1662841(2000b:65007)

[8] J. Lyness, Notes on lattice rules. Numerical integration and its complexity (Oberwolfach, 2001), J. Complexity 19 (2003), 321-331. MR1984117 (2004d:65035)

[9] J. Matoušek, Geometric Discrepancy, Algorithms and Combinatorics 18, Springer-Verlag, Berlin, 1999. MR1697825 (2001a:11135)

[10] H. Niederreiter, Random Number Generation and Quasi-Monte Carlo Methods, SIAM, Philadelphia, 1992. MR.1172997 (93h:65008)

[11] E. Novak and H. Woźniakowski, Tractability of Multivariate Problems, Volume I: Linear Information, EMS, Zürich, 2008. MR 2455266 (2009m:46037)

[12] D. Nuyens and R. Cools, Fast algorithms for component-by-component construction of rank1 lattice rules in shift-invariant reproducing kernel Hilbert spaces. Math. Comp. 75 (2006), 903-920. MR2196999 (2007a:65032)

[13] N. N. Osipov, Construction of lattice cubature formulas with the trigonometric $d$-property on the basis of extremal lattices. (Russian) Zh. Vychisl. Mat. Mat. Fiz. 48 (2008), 212-219. MR2426458 (2009e:65048)

[14] I. H. Sloan and S. Joe, Lattice Methods for Multiple Integration, Clarendon Press, Oxford, 1994. MR1442955(98a:65026)

[15] I. H. Sloan, F. Y. Kuo, and S. Joe, Constructing randomly shifted lattice rules in weighted Sobolev spaces. SIAM J. Numer. Anal. 40 (2002), 1650-1665. MR1950616 (2003m:65031)

[16] I. H. Sloan and H. Woźniakowski, An intractability result for multiple integration. Math. Comp. 66 (1997), 1119-1124. MR1401946 (97i:41040)

[17] I. H. Sloan and H. Woźniakowski, When are quasi-Monte Carlo algorithms efficient for high dimensional integrals? J. Complexity 14 (1998), 1-33. MR1617765 (99d:65384)

[18] I. H. Sloan and H. Woźniakowski, Tractability of multivariate integration for weighted Korobov classes. J. Complexity 17 (2001), 697-721. MR1881665 (2003g:65030)

School of Mathematics and Statistics, The University of New South Wales, Sydney, NSW 2052, Australia

E-mail address: josef.dick@unsw.edu.au

Institut für Finanzmathematik, Universität Linz, Altenbergstrasse 69, A-4040 Linz, Austria

E-mail address: gerhard.larcher@jku.at

Institut für Finanzmathematik, Universität Linz, Altenbergstrasse 69, A-4040 Linz, Austria

E-mail address: friedrich.pillichshammer@jku.at

Department of Computer Science, Columbia University, New York, New York 10027, USA and Institute of Applied Mathematics, University of Warsaw, ul. Banacha 2, 02097 WarszaWa, Poland

E-mail address: henryk@cs.columbia.edu 